\title{
Faster Carry Bit Computation for Adder Circuits with Prescribed Arrival Times
}

\author{
Ulrich Brenner, Anna Hermann \\ Research Institute for Discrete Mathematics, University of Bonn
}

\begin{abstract}
We consider the fundamental problem of constructing fast circuits for the carry bit computation in binary addition. Up to a small additive constant, the carry bit computation reduces to computing an AND-OR path, i.e., a formula of type $t_{0} \wedge\left(t_{1} \vee\left(t_{2} \wedge\left(\ldots t_{m-1}\right) \ldots\right)\right.$ or $t_{0} \vee\left(t_{1} \wedge\left(t_{2} \vee\left(\ldots t_{m-1}\right) \ldots\right)\right.$. We present an algorithm that computes the fastest known Boolean circuit for an AND-OR path with given arrival times $a\left(t_{0}\right), \ldots, a\left(t_{m-1}\right)$ for the input signals. Our objective function is delay, a natural generalization of depth with respect to arrival times. The maximum delay of the circuit we compute is $\log _{2} W+\log _{2} \log _{2} m+$ $\log _{2} \log _{2} \log _{2} m+4.3$, where $W:=\sum_{i=0}^{m-1} 2^{a\left(t_{i}\right)}$. Note that $\left\lceil\log _{2} W\right\rceil$ is a lower bound on the delay of any circuit depending on inputs $t_{0}, \ldots, t_{m-1}$ with prescribed arrival times. Our method yields the fastest circuits for AND-OR paths, carry bit computation and adders in terms of delay known so far.
\end{abstract}

\section{Introduction}

An AND-OR path is a Boolean formula of type

$$
t_{0} \wedge\left(t _ { 1 } \vee ( t _ { 2 } \wedge ( \ldots t _ { m - 1 } ) \ldots ) \quad \text { or } \quad t _ { 0 } \vee \left(t_{1} \wedge\left(t_{2} \vee\left(\ldots t_{m-1}\right) \ldots\right)\right.\right.
$$

We assume that for each Boolean input variable $t_{i}(i \in\{0, \ldots, m-1\})$, an arrival time $a\left(t_{i}\right) \in \mathbb{N}$ is given. Our goal is to find a Boolean circuit using only AND and OR gates that computes the Boolean function of a given AND-OR path and minimizes the maximum delay of the inputs. Here, the delay of an input $t_{i}$ in a Boolean circuit is its arrival time $a\left(t_{i}\right)$ plus the length of a maximum directed path in the circuit starting at $t_{i}$. Thus, the concept of delay minimization generalizes the concept of depth minimization. When only depth is considered, one has to assume that all input signals are available at the same time (uniform arrival times), which is not the case on real-world chips. Hence, taking non-uniform arrival times into account leads to a much more realistic problem formulation.

\subsection{Applications of And-Or Path Optimization}

Realizing AND-Or paths with fast circuits is important for VLSI design in different aspects.

Computing fast AND-OR paths is, up to a small additive constant, equivalent to constructing fast adder circuits. To see this, suppose we want to compute the sum of two binary numbers $x=\left(x_{r-1}, \ldots, x_{0}\right)$ and $y=\left(y_{r-1}, \ldots, y_{0}\right)$ with most significant bit $r-1$. Starting with $c_{1}=$ 


\begin{tabular}{llll} 
Author & Upper bound on delay & Size & Maximum fanout \\
\hline$[15] ;[7]$ & $1.441 \log _{2} W+2.674$ & $\mathcal{O}(m)$ & 2 \\
{$[14] ;[18]$} & $(1+\varepsilon)\left\lceil\log _{2} W\right\rceil+\frac{3}{\varepsilon}+5$ & $\mathcal{O}\left(\frac{m}{\varepsilon}\right)$ & 2 \\
{$[14] ;[18]$} & $(1+\varepsilon)\left\lceil\log _{2} W\right\rceil+\frac{3}{\varepsilon}+5$ & $\mathcal{O}(m)$ & $2^{\frac{1}{\varepsilon}}$ \\
{$[18]$} & $\left\lceil\log _{2} W\right\rceil+2 \sqrt{2 \log _{2} m-2}+6$ & $\mathcal{O}\left(m \sqrt{\log _{2} m}\right)$ & 2 \\
{$[18]$} & $\left\lceil\log _{2} W\right\rceil+2 \sqrt{2 \log _{2} m-2}+6$ & $\mathcal{O}(m)$ & $2 \sqrt{2 \log _{2} m-2}+1$ \\
Here & $\log _{2} W+\log _{2} \log _{2} m$ & $\mathcal{O}\left(m \log _{2} m \log _{2} \log _{2} m\right)$ & $\mathcal{O}\left(\log _{2} m\right)$ \\
& $+\log _{2} \log _{2} \log _{2} m+4.3$ & & \\
\hline
\end{tabular}

Table 1: Known upper bounds on delay of AND-OR paths with non-uniform arrival times.

$\left(x_{0} \wedge y_{0}\right)$, the carry bits can be computed recursively via

$$
\begin{aligned}
c_{i+1} & =\left(x_{i} \wedge y_{i}\right) \vee\left(\left(x_{i} \vee y_{i}\right) \wedge c_{i}\right) \\
& =\underbrace{\left(x_{i} \wedge y_{i}\right)}_{t_{0}} \vee(\underbrace{\left(x_{i} \vee y_{i}\right)}_{t_{1}} \wedge(\underbrace{\left(x_{i-1} \wedge y_{i-1}\right)}_{t_{2}} \vee(\underbrace{\left(x_{i-1} \vee y_{i-1}\right)}_{t_{3}} \wedge \ldots)))
\end{aligned}
$$

for $0<i<r$. The computation of the $i$ th carry bit is hence essentially the evaluation of an AND-OR path of length $2 i$. Once all carry bits are known, the sum can be computed with an additional delay of 2 . Adders with non-uniform input arrival times occur, e.g., as a part of multiplication units (see Zimmermann [21]). Depth optimization of adders (and thus of AND-OR paths for the carry bit computation) is a classical and well-studied optimization problem, see Sklansky [17], Brent [1], Khrapchenko [9], Kogge and Stone [11], Ladner and Fischer [13], and Brent and Kung [2].

Another application of AND-OR path optimization is the comparison of binary numbers since lexicographic comparison can be expressed as an AND-Or path (see, e.g., Grinchuk [6]).

More generally, AND-OR path optimization is used to speed up timing-critical paths on VLSI chips. Note that the most critical path $P$ on a chip can be decomposed into, e.g., AND gates and inverter gates. Using De Morgan's laws, the inverters can be pushed to the inputs of $P$, making $P$ a path consisting of AND and OR gates (not necessarily alternating). In [20], Werber et al. successfully applied their AND-OR path optimization algorithm presented in [15] for iteratively improving such paths in a late stage of physical design. In this context, it is essential that the objective function of the algorithm used is delay instead of depth since typically, input signals of the most critical path will not arrive simultaneously.

\subsection{Previous Work}

For AND-OR paths with uniform arrival times (i.e., only depth is considered), the currently fastest Boolean circuit has been proposed by Grinchuk [6] reaching a depth of $\log _{2} m+\log _{2} \log _{2} m+\mathcal{O}(1)$. This is close to the best known lower bounds on depth: Khrapchenko [10] showed that any circuit for an AND-OR path has a depth of at least $\log _{2} m+0.15 \log _{2} \log _{2} \log _{2} m+\Theta(1)$. The result is based on a lower bound of $\Theta\left(\frac{m \log _{2} m \log _{2} \log _{2} m}{\log _{2} \log _{2} \log _{2} \log _{2} m}\right)$ on the product of size and depth of a Boolean formula for an AND-OR path (see Commentz-Walter and Sattler [4]). For monotone circuits, i.e., circuits without negations (and the circuit built in [6] is a monotone circuit), this lower bound can be improved to $\Theta\left(m \log _{2}^{2} m\right)$ (see Commentz-Walter [3]). This directly implies a lower bound of $\log _{2} m+\log _{2} \log _{2} m+\Theta(1)$ on the depth of a monotone circuit for an AND-OR path. 
For AND-OR paths with non-uniform arrival times $a\left(t_{0}\right), \ldots, a\left(t_{m-1}\right)$, the value $\left\lceil\log _{2} W\right\rceil$ is a lower bound on the achievable delay, where $W:=\sum_{i=0}^{m-1} 2^{a\left(t_{i}\right)}$ (see, e.g., Rautenbach et al. [15]). No stronger lower bounds on delay are known. Rautenbach et al. [15] presented an algorithm computing a Boolean circuit for an AND-OR path with delay at most $1.441 \log _{2} W+3$. This delay bound was improved to $1.441 \log _{2} W+2.674$ by Held and Spirkl [7]. In both of these circuits, socalled 2-input prefix gates are used, and it can be shown that any AND-OR path realization based on prefix gates has a delay of at least $\log _{\varphi}\left(\sum_{i=0}^{m-1} \varphi^{a\left(t_{i}\right)}\right)$ where $\varphi=\frac{1+\sqrt{5}}{2} \approx 1.618$ is the golden ratio (see [7]). In particular, this implies that any prefix-based AND-OR path realization has a depth of at least $1.44 \log _{2} m-1$. Without using prefix gates, Rautenbach et al. [14] presented a circuit for an AND-OR path with delay at most $(1+\varepsilon)\left\lceil\log _{2} W\right\rceil+c_{\varepsilon}$ (for any $\varepsilon>0$ ), where $c_{\varepsilon}$ is a number depending on $\varepsilon$, only. Spirkl [18] specified the delay bound to $(1+\varepsilon)\left\lceil\log _{2} W\right\rceil+\frac{6}{\varepsilon}+8+5 \varepsilon$ and improved it to $(1+\varepsilon)\left\lceil\log _{2} W\right\rceil+\frac{3}{\varepsilon}+5$. Moreover, Spirkl [18] described a circuit with a delay of at most $\left\lceil\log _{2} W\right\rceil+2 \sqrt{2 \log _{2} m-2}+6$. Note that for any $\varepsilon>0$, this is actually a better delay bound than $(1+\varepsilon)\left\lceil\log _{2} W\right\rceil+\frac{3}{\varepsilon}+5$ (because $\varepsilon \log _{2} W+\frac{3}{\varepsilon}+5 \geq 2 \sqrt{3 \log _{2} W}+5 \geq$ $2 \sqrt{3 \log _{2} m}+5 \geq 2 \sqrt{2 \log _{2} m-2}+6$ ). Up to now, this is the fastest circuit for AND-Or paths with non-uniform arrival times. Table 1 summarizes these results in comparison with our delay bound. We also state size (i.e., number of gates) and maximum fanout of the constructed circuits. Note that some methods trade off size against fanout and provide two different circuits.

All the AND-OR path circuits presented in the table can be used directly to obtain adder circuits with the same delays by computing each carry bit separately. However, the size of the arising circuits would be super-quadratic. The construction of linear-size adder circuits with our delay guarantee is part of ongoing work.

\subsection{Our Contribution}

In this paper, we present an algorithm with running time $\mathcal{O}\left(m^{2} \log _{2} m\right)$ that computes a Boolean circuit for AND-OR paths (with $m \geq 3$ ) using only two-input AND and OR gates with a delay of at most

$$
\log _{2} W+\log _{2} \log _{2} m+\log _{2} \log _{2} \log _{2} m+4.3,
$$

size $\mathcal{O}\left(m \log _{2} m\right)$ and maximum fanout $\log _{2} m+\log _{2} \log _{2} m+\log _{2} \log _{2} \log _{2} m+3.3$. In terms of delay, this yields the currently best known circuits for AND-OR paths and thus also adder circuits. In particular, we improve the previously best known delay bound of $\left\lceil\log _{2} W\right\rceil+2 \sqrt{2 \log _{2} m-2}+6$ by Spirkl [18] for each $m \geq 3$ as well as asymptotically. The construction of the circuit is based on a recursive approach similar to the algorithm of Grinchuk [6] for uniform arrival times.

The rest of the paper is organized as follows. In Section 2, we introduce basic definitions and results. We give a formal description of the problem (Subsection 2.1), define splitting steps which allow us to partition an instance into smaller sub-instances (Subsection 2.2) and introduce a measure for deciding which instances admit an AND-OR path realization with a given delay (Subsection 2.3). Section 3 classifies these instances, which is the major step of the paper. In Section 4, we deduce how to construct circuits realizing AND-OR paths with a delay of at most $\log _{2} W+\log _{2} \log _{2} m+\log _{2} \log _{2} \log _{2} m+4.3$ and analyze the size and fanout as well as the runtime needed to compute such circuits. In Section 5, we extend this result to paths that also consist of AND and OR gates only, but not necessarily alternatingly. 


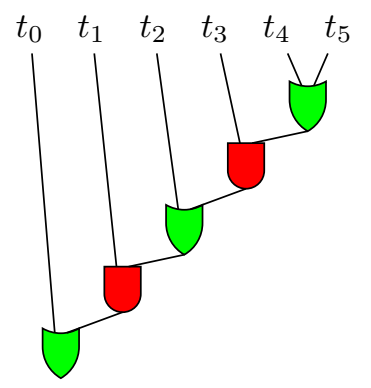

(a) AND-OR path $g^{*}\left(\left(t_{0}, \ldots, t_{5}\right)\right)$.

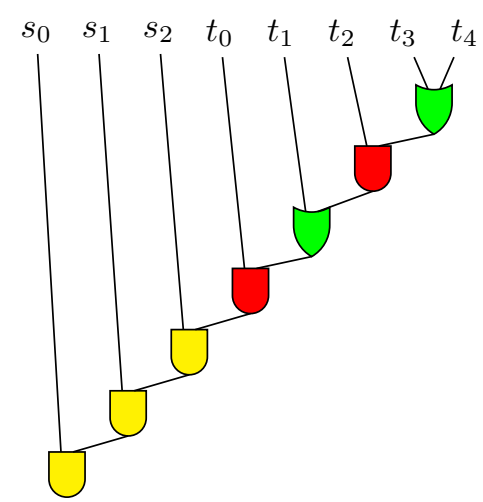

(b) Extended AND-OR path $f\left(\left(s_{0}, s_{1}, s_{2}\right),\left(t_{0}, \ldots, t_{4}\right)\right)$.

Figure 1: Examples for (extended) AND-OR paths.

\section{Preliminaries}

\subsection{Problem Formulation}

We denote the set of natural numbers including zero by $\mathbb{N}$. Our notation regarding Boolean functions and circuits is based on Savage [16]. Given $r \in \mathbb{N}$ and a Boolean function $h:\{0,1\}^{r} \rightarrow$ $\{0,1\}$ with Boolean input variables (shorter, inputs) $x_{0}, \ldots, x_{r-1}$, we write $x=\left(x_{0}, \ldots, x_{r-1}\right)$ as a shorthand for all inputs with fixed ordering. If $r=0$, we write $x=()$.

Definition 1. Let Boolean input variables $t=\left(t_{0}, \ldots, t_{m-1}\right)$ for some $m \in \mathbb{N}$ with $m>0$ be given. We call each of the recursively defined functions

$$
g(t)=\left\{\begin{array}{ll}
t_{0} & m=1 \\
t_{0} \wedge g^{*}\left(\left(t_{1}, \ldots, t_{m-1}\right)\right) & m>1
\end{array} \quad \text { and } \quad g^{*}(t)= \begin{cases}t_{0} & m=1 \\
t_{0} \vee g\left(\left(t_{1}, \ldots, t_{m-1}\right)\right) & m>1\end{cases}\right.
$$

an And-Or path on $m$ inputs.

We want to realize a given AND-OR path as a Boolean circuit over the basis $\{\wedge, \vee\}$. A Boolean circuit over the basis $\{\wedge, \vee\}$ is a directed acyclic graph such that

- the nodes with indegree 0, called inputs, are labeled by Boolean variables,

- there is only one node with outdegree 0 , called output, and it has indegree exactly 1 ,

- each of the remaining nodes has indegree exactly 2 and outdegree at least 1 and is labeled either as an AND gate or as an OR gate.

The logical function on the input variables computed by the output of the circuit can be obtained by combining the logical functions represented by the gates recursively. A circuit is a realization of the AND-OR path $g(t)$ if and only if the output signal equals $g(t)$ for all input values (correspondingly for $\left.g^{*}\right)$. Figure 1 (a) shows a Boolean circuit which is a straightforward realization of the AND-OR path $g^{*}\left(\left(t_{0}, \ldots, t_{5}\right)\right)$. We omit drawing the output in such pictures - the unique predecessor of the output will always be the bottommost gate.

We assume that each input variable is associated with a prescribed arrival time. 
Definition 2. Let $r \in \mathbb{N}$ and a Boolean function $h:\{0,1\}^{r} \rightarrow\{0,1\}$ on Boolean input variables $x=\left(x_{0}, \ldots, x_{r-1}\right)$ with arrival times $a:\left\{x_{0}, \ldots, x_{r-1}\right\} \rightarrow \mathbb{N}$ be given. Consider a circuit $C$ computing $h$. For $i=0, \ldots, r-1$, the delay of input $x_{i}$ is defined as $a\left(x_{i}\right)+l\left(x_{i}\right)$, where $l\left(x_{i}\right)$ denotes the maximum number of gates of any directed path in $C$ starting at input $x_{i}$. The delay of the circuit $C$ is the maximum delay of any input.

Note that for uniform arrival times, e.g., $a \equiv 0$, the delay of a circuit is simply the depth of the circuit.

Our goal is computing fast circuits for AND-OR paths, i.e., solving the following problem:

Problem 3 (And-OR Path Optimization Problem). For $m \in \mathbb{N}$ with $m>0$, consider Boolean input variables $t=\left(t_{0}, \ldots, t_{m-1}\right)$ with arrival times $a:\left\{t_{0}, \ldots, t_{m-1}\right\} \rightarrow \mathbb{N}$. Find circuits over the basis $\{\wedge, \vee\}$ that compute the AND-OR paths $g(t)$ and $g^{*}(t)$ with minimum possible delay.

Note that adding the same constant to all arrival times does not change the problem. This is why we only allow non-negative arrival times in this formulation. Moreover, forbidding nonintegral arrival times is not a significant restriction because rounding up all arrival times will change the delay of any circuit by less than 1 . Therefore, we only consider natural numbers as arrival times.

\subsection{Recursive Circuit Construction}

We construct fast circuits for AND-OR paths in a recursive way. Before describing all details of the approach, we explain the idea of the induction step.

Suppose we want to realize the AND-OR path $g^{*}(t)=t_{0} \vee\left(t_{1} \wedge t_{2} \vee\left(t_{3} \wedge\left(\ldots t_{m-1} \ldots\right)\right)\right)$. We subdivide the inputs $t_{0}, \ldots, t_{m-1}$ into two groups $t_{0}, \ldots, t_{2 k}$ and $t_{2 k+1}, \ldots, t_{m-1}$. Recursively, we compute fast circuits for the AND-OR paths on each of these input sets. These two circuits can be combined to a circuit for the whole AND-OR path as illustrated in an example with $m=12$ and $k=3$ in Figure 2; the general construction is described in Lemma 5 . The output of the circuit for the subinstance $t_{2 k+1}, \ldots, t_{m-1}$ is combined with every second input of $t_{0}, \ldots, t_{2 k}$ by using only AND gates. Just one additional OR gate (labeled " $G$ " in the picture) is needed to compute a circuit for the whole AND-OR path. It is not too difficult to check that the circuits in Figure 2 (a) and (b) are logically equivalent. To see this, note that the output of the circuit in (a) is "true" if and only if there is an $i \in\{0,2,4,6,8,10,11\}$ such that the input signals at $t_{i}$ and at every $t_{j}$ with $j$ odd and $j \in\{1, \ldots, i-1\}$ are "true". This is also a sufficient and necessary condition for a "true" as an output of the circuit in (b).

Note that while the left input of gate $G$ in the example is the output of an AnD-Or path, the right input of $G$ is the output of a function combining the AND-OR path for $t_{2 k+1}, \ldots, t_{m-1}$ with a multiple-input AND. The occurrence of such functions in our recursion requires generalizing the concept of AND-OR paths.

Definition 4. Given $n, m \in \mathbb{N}, m>0$, and inputs $s_{0}, \ldots, s_{n-1}, t_{0}, \ldots, t_{m-1}$ subdivided into $s=\left(s_{0}, \ldots, s_{n-1}\right)$ and $t=\left(t_{0}, \ldots, t_{m-1}\right)$, we define the extended And-Or paths

$$
f(s, t)=s_{0} \wedge f\left(\left(s_{1}, \ldots, s_{n-1}\right), t\right) \quad \text { and } \quad f^{*}(s, t)=s_{0} \vee f^{*}\left(\left(s_{1}, \ldots, s_{n-1}\right), t\right),
$$

where $f(s, t)=g(t), f^{*}(s, t)=g^{*}(t)$ in the case that $s=()$. We call the input variables $s$ symmetric inputs and the input variables $t$ alternating inputs, respectively.

Figure 1 (b) shows the extended AND-OR path $f(s, t)$ for $s=\left(s_{0}, s_{1}, s_{2}\right)$ and $t=\left(t_{0}, \ldots, t_{4}\right)$. We shall always assume that the set of input variables contained in $s$ and $t$ are disjoint sets 


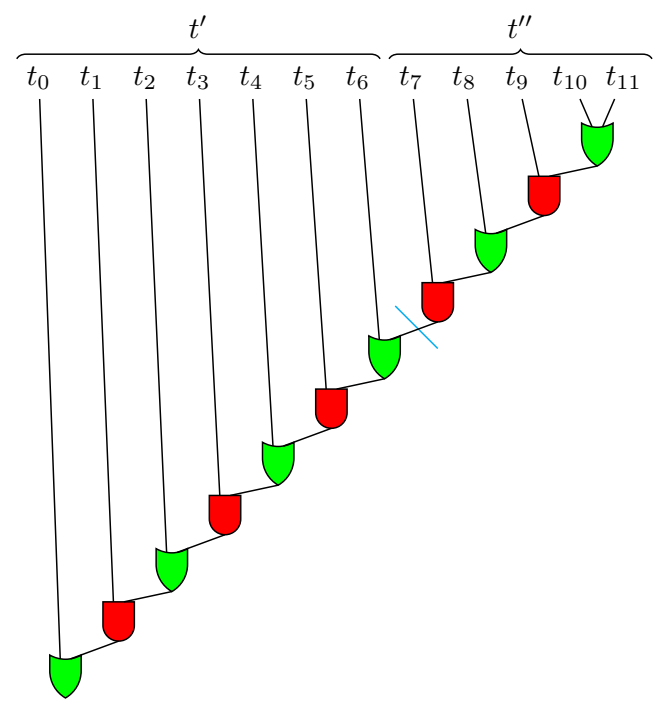

(a) And-Or path on $t=\left(t_{0}, \ldots, t_{11}\right)$.

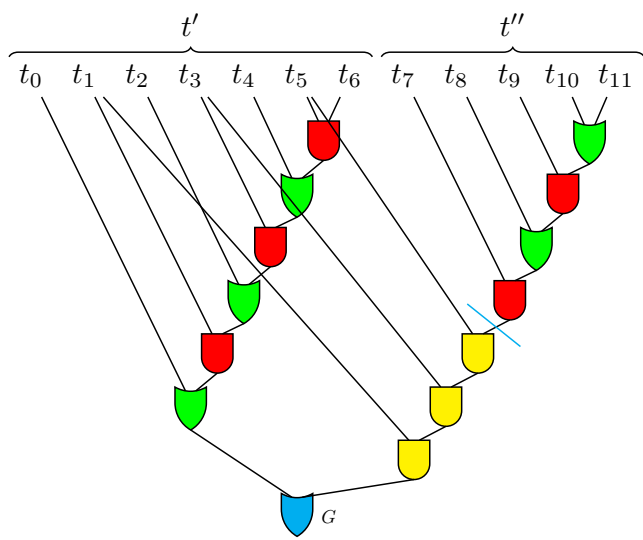

(b) Alternating split with $t^{\prime}=\left(t_{0}, \ldots, t_{6}\right)$.

Figure 2: Performing the alternating split on $g^{*}\left(\left(t_{0}, \ldots, t_{11}\right)\right)=t_{0} \vee\left(t_{1} \wedge\left(\ldots\left(t_{10} \vee t_{11}\right) \ldots\right)\right)$.

indexed by $s_{0}, \ldots, s_{n-1}$ and $t_{0}, \ldots, t_{m-1}$. Note that expanding the definitions of $f(s, t)$ and $f^{*}(s, t)$ given in Definition 4 yields

$$
\begin{aligned}
f(s, t) & =s_{0} \wedge \ldots \wedge s_{n-1} \wedge g(t)=s_{0} \wedge \ldots \wedge s_{n-1} \wedge t_{0} \wedge\left(t_{1} \vee t_{2} \wedge\left(t_{3} \vee\left(\ldots t_{m-1} \ldots\right)\right)\right), \\
f^{*}(s, t) & =s_{0} \vee \ldots \vee s_{n-1} \vee g^{*}(t)=s_{0} \vee \ldots \vee s_{n-1} \vee t_{0} \vee\left(t_{1} \wedge t_{2} \vee\left(t_{3} \wedge\left(\ldots t_{m-1} \ldots\right)\right)\right),
\end{aligned}
$$

where, for $m$ odd, the innermost operation of $f(s, t)$ and $f^{*}(s, t)$ is $\vee$ and $\wedge$, respectively, and vice versa for $m$ even.

Due to the duality principle of Boolean algebra, over the basis $\{\wedge, \vee\}$, any realization for $f(s, t)$ yields a realization of $f^{*}(s, t)$ and vice versa by switching all AND and OR gates. In order to compute fast realizations for $f$ and $f^{*}$, we will apply two methods that allow realizing $f$ and $f^{*}$ recursively. Each of these methods reduces the problem of realizing $f(s, t)$ to the problem of realizing extended AND-OR paths with strictly fewer symmetric or alternating inputs.

First, we formally describe the well-known method which is depicted in Figure 2 for the special case that $s=(), m=12$ and $k=3$.

Lemma 5. Let input variables $s$ and $t$ and an integer $k$ with $0 \leq k<\frac{m-1}{2}$ be given. Denote by $t^{\prime}$ the (odd-length) prefix $t^{\prime}=\left(t_{0}, t_{1}, \ldots, t_{2 k}\right)$ of $t$, and by $t^{\prime \prime}$ the remaining inputs of $t$, i.e., $t^{\prime \prime}=\left(t_{2 k+1}, \ldots, t_{m-1}\right)$. Then, we have

$$
f^{*}(s, t)=f^{*}\left(s, t^{\prime}\right) \vee f\left(\widehat{t^{\prime}}, t^{\prime \prime}\right)
$$

where $\widehat{t^{\prime}}:=\left(t_{1}^{\prime}, t_{3}^{\prime}, t_{5}^{\prime}, \ldots, t_{2 k-1}^{\prime}\right)$ contains every second entry of $t^{\prime}$.

Proof. At first, we prove the following claim:

Claim: For any Boolean variable $x$, we have

$$
g^{*}\left(\left(t_{0}, \ldots, t_{2 k}, x\right)\right)=g^{*}\left(\left(t_{0}, \ldots, t_{2 k}\right)\right) \vee\left(t_{1} \wedge t_{3} \wedge \ldots \wedge t_{2 k-1} \wedge x\right)
$$


Proof of the claim: We prove the claim by induction on $k$. For $k=0$, we have

$$
g^{*}\left(\left(t_{0}, \ldots, t_{2 k}, x\right)\right)=g^{*}\left(\left(t_{0}, x\right)\right)=t_{0} \vee x=g^{*}\left(\left(t_{0}, \ldots, t_{2 k}\right)\right) \vee\left(t_{1} \wedge t_{3} \wedge \ldots \wedge t_{2 k-1} \wedge x\right) .
$$

Assuming statement (2) holds for some $k \geq 0$ and setting $x_{1}:=t_{2 k+1} \wedge\left(t_{2 k+2} \vee x\right)$ and $x_{2}:=$ $t_{2 k+1} \wedge t_{2 k+2}$, we compute

$$
\begin{array}{ll} 
& g^{*}\left(\left(t_{0}, \ldots, t_{2(k+1)}, x\right)\right) \\
\stackrel{\text { Def. }}{=} & g^{*}\left(\left(t_{0}, \ldots, t_{2 k}, x_{1}\right)\right) \\
\stackrel{(2)}{=} & g^{*}\left(\left(t_{0}, \ldots, t_{2 k}\right)\right) \vee\left(t_{1} \wedge t_{3} \wedge \ldots \wedge t_{2 k-1} \wedge x_{1}\right) \\
= & g^{*}\left(\left(t_{0}, \ldots, t_{2 k}\right)\right) \vee\left(t_{1} \wedge t_{3} \wedge \ldots \wedge t_{2 k-1} \wedge t_{2 k+1} \wedge\left(t_{2 k+2} \vee x\right)\right) \\
= & g^{*}\left(\left(t_{0}, \ldots, t_{2 k}\right)\right) \vee\left(t_{1} \wedge t_{3} \wedge \ldots \wedge t_{2 k-1} \wedge x_{2}\right) \vee\left(t_{1} \wedge t_{3} \wedge \ldots \wedge t_{2 k-1} \wedge t_{2 k+1} \wedge x\right) \\
\stackrel{(2)}{=} & g^{*}\left(\left(t_{0}, \ldots, t_{2 k}, x_{2}\right)\right) \vee\left(t_{1} \wedge t_{3} \wedge \ldots \wedge t_{2 k-1} \wedge t_{2 k+1} \wedge x\right) \\
\stackrel{\text { Def. }}{=} 1 & g^{*}\left(\left(t_{0}, \ldots, t_{2 k+2}\right)\right) \vee\left(t_{1} \wedge t_{3} \wedge \ldots \wedge t_{2 k-1} \wedge t_{2 k+1} \wedge x\right) .
\end{array}
$$

This proves the inductive step and thus the claim.

From this claim and Definition 1, we conclude

$$
\begin{array}{rll}
f^{*}(s, t) & \stackrel{\text { Def. }}{=}{ }^{4} & s_{0} \vee \ldots \vee s_{n-1} \vee g^{*}(t) \\
& \stackrel{\text { Def. } 1}{=} & s_{0} \vee \ldots \vee s_{n-1} \vee g^{*}\left(\left(t_{0}, \ldots, t_{2 k}, g\left(t^{\prime \prime}\right)\right)\right) \\
& \stackrel{(2)}{=} & s_{0} \vee \ldots \vee s_{n-1} \vee g^{*}\left(\left(t_{0}, \ldots, t_{2 k}\right)\right) \vee\left(t_{1} \wedge t_{3} \wedge \ldots \wedge t_{2 k-1} \wedge g\left(t^{\prime \prime}\right)\right) \\
& \stackrel{\text { Def. } 4}{=} & f^{*}\left(s, t^{\prime}\right) \vee f\left(\widehat{t}^{\prime}, t^{\prime \prime}\right) .
\end{array}
$$

The previous lemma allows us to recursively construct a circuit for $f^{*}(s, t)$ : Inductively, we can assume that we can compute a fast circuit for $f^{*}\left(s, t^{\prime}\right)$ and $f\left(\widehat{t}^{\prime}, t^{\prime \prime}\right)$, respectively. Combining these circuits as in equation (1) yields a good circuit for $f^{*}(s, t)$. We call this way to construct a circuit for $f^{*}(s, t)$ an alternating split.

Similarly, if $n>0$, the definition of $f(s, t)$ (see Definition 4) yields two symmetric splits indicated by the equations

$$
\begin{aligned}
& f(s, t)=\left(s_{0} \wedge \ldots \wedge s_{n-1}\right) \wedge g(t) \\
& f(s, t)=\left(s_{0} \wedge \ldots \wedge s_{n-1} \wedge t_{0}\right) \wedge g^{*}\left(\left(t_{1}, \ldots, t_{m-1}\right)\right) .
\end{aligned}
$$

Here, we only inquire a recursive circuit construction for the arising AND-OR path since a delay-optimum symmetric AND-tree can be constructed via Huffman coding [8] as explained in Remark 8.

Note that dualizing the splits (1), (3) and (4) yields analogous splits for $f^{*}$ :

$$
\begin{aligned}
f(s, t) & =f\left(s, t^{\prime}\right) \wedge f^{*}\left(\widehat{t^{\prime}}, t^{\prime \prime}\right) \\
f^{*}(s, t) & =\left(s_{0} \vee \ldots \vee s_{n-1}\right) \vee g^{*}(t) \\
f^{*}(s, t) & =\left(s_{0} \vee \ldots \vee s_{n-1} \vee t_{0}\right) \vee g\left(\left(t_{1}, \ldots, t_{m-1}\right)\right)
\end{aligned}
$$

\subsection{Delay and Weight}

We will realize extended AND-OR paths with good delay using the recursive methods defined in Subsection 2.2. For this, we need to classify inputs by their weight. 
Definition 6. Given inputs $x=\left(x_{0}, \ldots, x_{r-1}\right)$ and arrival times $a$, for $i=0, \ldots, r-1$, the weight of input $x_{i}$ is $W\left(x_{i}\right):=2^{a\left(x_{i}\right)}$, and the weight of $x$ is $W(x):=\sum_{i=0}^{r-1} W\left(x_{i}\right)$.

Note that in this definition, the weights $W\left(x_{i}\right)$ and $W(x)$ do not only depend on the inputs, but also on the input arrival times.

Remark 7. By Definition 6 , we have $W\left(x_{i}\right) \geq 1$ for any input $x_{i}$ with $a\left(x_{i}\right) \geq 0$.

The definition of the weight is motivated by the fact that for symmetric binary functions, i.e., AND trees or OR trees, the optimum delay achievable for any realization can be derived from the weight of the inputs directly.

Remark 8. A binary tree on inputs $x=\left(x_{0}, \ldots, x_{r-1}\right)$ with weight $W(x)$ can be realized with delay $d$ if and only if Kraft's inequality $W(x) \leq 2^{d}$ is fulfilled [12], see also Golumbic [5]. A delay-optimum tree can be computed in runtime $\mathcal{O}\left(r \log _{2} r\right)$ via a greedy algorithm based on Huffman coding [8]. A short proof of this can be found in Werber [19].

Note that when $t$ has at most 2 entries, $f(s, t)$ is a symmetric binary AND tree.

Lemma 9. For Boolean input variables $s$ and $t$ with $m \leq 2$ and arrival times $a$, we can realize $f(s, t)$ with delay $d$ if and only if $W(s)+W(t) \leq 2^{d}$.

For the case that $t$ has more than 2 entries, we give an upper bound on the delay of $f(s, t)$ in Section 3.

\section{Bounding the Weight for Given Delay}

We will prove an upper bound on the delay of AND-Or paths by a reverse argument similarly as in Grinchuk's proof for the case of uniform input arrival times [6]. Grinchuk fixes a depth bound $d$ and the number $n$ of symmetric inputs $s$, and determines how many alternating inputs $t$ an AND-Or path may have such that $f(s, t)$ can be realized with depth $d$. Similarly, given symmetric inputs $s$ with a fixed weight $w$ and a fixed delay bound $d$, we will determine for which alternating inputs $t$ a realization for $f(s, t)$ with delay $d$ can be guaranteed. Since it is difficult to classify these $t$ exactly, we distinguish different alternating inputs $t$ by their weight only.

In order facilitate the formulation of our main statement, we fix a constant $\zeta$, see also Remark 12.

Definition 10. Let $\zeta:=1.9$ be a fixed constant.

We aim at proving the following statement:

Theorem 11. Let $d, w \in \mathbb{N}$ with $d>1$ and $0 \leq w<2^{d-1}$ be given. Consider Boolean input variables $s$ and $t$ with $W(s)=w$ and

$$
W(t) \leq \zeta \frac{2^{d-1}-w}{d \log _{2}(d)} .
$$

Then, there is a circuit realizing $f(s, t)$ with delay at most $d$.

We will prove Theorem 11 by induction on $d$. Based on this, we will deduce the desired upper bound of $\log _{2} W+\log _{2} \log _{2} m+\log _{2} \log _{2} \log _{2} m+4.3$ on the delay of AND-OR paths in Section 4 . 
Remark 12. Note that the choice of the constant $\zeta$ influences the additive constant (here 4.3) in the delay bound. For example, if we chose $\zeta:=1$, we would need to replace the additive constant by 5 . On the other hand, the higher $\zeta$, the larger the minimum value of $d$ is for which the induction step works, which increases the proof complexity for the base case.

Most parts of the proof of Theorem 11 will work for any $\zeta$ with $1 \leq \zeta<2$; only at the end of the proof of Lemma 23 we demand $\zeta \leq 1.9$. A slightly larger choice of $\zeta$ would be possible, but using $\zeta=1.9$ keeps calculations simpler. An improvement of the additive term to, e.g., 4.2 would only be possible for $\zeta \geq 1.992$, for which we cannot prove Theorem 11 .

For proving Theorem 11, we would like to proceed by induction on $d$ making use of the restructuring formulas presented in Section 2.2. The following remark explains why the inductive step from $d$ to $d+1$ does not work directly.

Remark 13. Suppose that Theorem 11 holds for some $d>1$ and all $0 \leq w<2^{d-1}$. In order to prove Theorem 11 for $d+1$, we need to show that, given input variables $s$ and $t$ with $0 \leq w:=W(s)<2^{d}$ and $W(t) \leq \zeta \frac{2^{d}-w}{(d+1) \log _{2}(d+1)}, f(s, t)$ can be realized with delay $d+1$. In the case that $w<2^{d-1}$, we would like to apply the alternating split $f(s, t)=f\left(s, t^{\prime}\right) \wedge f^{*}\left(\widehat{t^{\prime}}, t^{\prime \prime}\right)$ given by (5). If we choose the prefix $t^{\prime}$ of $t$ such that

$$
W\left(t^{\prime}\right) \leq \zeta \frac{2^{d-1}-w}{d \log _{2}(d)}
$$

(whenever this is possible), the induction hypothesis and the assumption $w<2^{d-1}$ allow us to construct a circuit for $f\left(s, t^{\prime}\right)$ with delay $d$. Thus, in order to construct a circuit with delay $d+1$ for $f(s, t)$, it remains to prove that $f^{*}\left(\widehat{t^{\prime}}, t^{\prime \prime}\right)$ admits a circuit with delay $d$. Again, by induction hypothesis, we need to show that $W\left(t^{\prime \prime}\right) \leq \zeta \frac{2^{d-1}-W\left(\widehat{t^{\prime}}\right)}{d \log _{2}(d)}$. But the only thing we know about $W\left(t^{\prime \prime}\right)$ is that

$$
W\left(t^{\prime \prime}\right)=W(t)-W\left(t^{\prime}\right) .
$$

Even if we choose the prefix $t^{\prime}$ maximal with (8), this will not give us a meaningful upper bound on $W\left(t^{\prime \prime}\right)$ since $W\left(t^{\prime}\right)$ might be arbitrarily small in comparison to $W(t)$.

Note that this is what distinguishes our proof from Grinchuk's [6]: For arrival times all 0, choosing $t^{\prime}$ maximal with (8) works well, since then, by maximality, we have $W\left(t^{\prime}\right)>\zeta \frac{2^{d-1}-w}{d \log _{2}(d)}-2$, hence equation (9) yields $W\left(t^{\prime \prime}\right)=W(t)-W\left(t^{\prime}\right)<W(t)-\zeta \frac{2^{d-1}-w}{d \log _{2}(d)}-2$. It turns out that this upper bound on $W\left(t^{* *}\right)$ suffices to prove that $f^{*}\left(\widehat{t^{\prime}}, t^{\prime \prime}\right)$ can be realized with delay $d$.

When arbitrary arrival times are present, a different proof idea is needed.

Thus, instead of proving Theorem 11 via induction on $d$, we strengthen the induction hypothesis and prove the stronger Theorem 15.

Definition 14. Let $m \in \mathbb{N}$ with $m>0$. For inputs $t=\left(t_{0}, \ldots, t_{m-1}\right)$ with arrival times $a$, we denote by $\Lambda_{t}$ the weight of the last two (or fewer) entries of $t$, i.e.,

$$
\Lambda_{t}:= \begin{cases}W\left(t_{0}\right), & m=1, \\ W\left(t_{m-2}\right)+W\left(t_{m-1}\right), & m>1 .\end{cases}
$$

Theorem 15. Let $d, w \in \mathbb{N}$ with $d>1$ and $0 \leq w<2^{d-1}$ be given. Consider Boolean input variables $s$ and $t$ with $W(s)=w$ and

$$
W(t) \leq \zeta \frac{2^{d-1}-w}{d \log _{2}(d)}+\frac{d-1}{d} \Lambda_{t}
$$

Then, there is a circuit realizing $f(s, t)$ with delay at most $d$. 
Note that since $\Lambda_{t} \geq 0$, Theorem 15 implies Theorem 11. The proof of Theorem 15 is the most important part of this paper and covers the rest of this section. First, we observe two ways to express requirement (10) differently.

Remark 16. Assuming the conditions of Theorem 15, the following statements are equivalent to requirement (10):

$$
\begin{array}{r}
\sum_{i=0}^{m-3} W\left(t_{i}\right)+\frac{\Lambda_{t}}{d} \leq \zeta \frac{2^{d-1}-w}{d \log _{2}(d)} \\
d \cdot \sum_{i=0}^{m-3} W\left(t_{i}\right)+\Lambda_{t} \leq \zeta \frac{2^{d-1}-w}{\log _{2}(d)}
\end{array}
$$

Statement (11) can be obtained from requirement (10) by subtracting $\frac{d-1}{d} \Lambda_{t}$. From this, multiplication with $d$ yields statement (12).

Next, we give an upper bound on the weight $W(t)+w$.

Lemma 17. Assuming the conditions of Theorem 15, we have

$$
W(t)+w \leq \begin{cases}2^{d-1} & \text { if } d \geq 2^{\zeta} \\ \frac{2^{d}}{\log _{2}(d)} & \text { otherwise }\end{cases}
$$

Proof. Using inequality (12), we obtain

$$
W(t)+w \stackrel{(12)}{\leq} \zeta \frac{2^{d-1}-w}{\log _{2}(d)}+w=\frac{\zeta 2^{d-1}+\left(\log _{2}(d)-\zeta\right) w}{\log _{2}(d)}
$$

If $d \geq 2^{\zeta}$, the condition $w<2^{d-1}$ yields

$$
W(t)+w \stackrel{(14)}{\leq} \frac{\zeta 2^{d-1}+\left(\log _{2}(d)-\zeta\right) w}{\log _{2}(d)} \leq \frac{2^{d-1}\left(\zeta+\log _{2}(d)-\zeta\right)}{\log _{2}(d)}=2^{d-1}
$$

Otherwise, if $d<2^{\zeta}$, the condition $w \geq 0$ yields

$$
W(t)+w \stackrel{(14)}{\leq} \frac{\zeta 2^{d-1}+\left(\log _{2}(d)-\zeta\right) w}{\log _{2}(d)} \leq \frac{\zeta 2^{d-1}}{\log _{2}(d)} \stackrel{\zeta<2}{<} \frac{2^{d}}{\log _{2}(d)} .
$$

The equivalent requirements (10), (11) and (12) as well as Lemma 17 will be used extensively when proving Theorem 15. For this proof, we proceed by induction on $d$. In Lemma 18, we will show as a base case that Theorem 15 holds for $d \leq 3$. Then, in Theorem 19, we will prove the inductive step: Assuming that Theorem 15 holds for some $d \geq 3$ and all $0 \leq w<2^{d-1}$, we will prove the statement for $d+1$ and all $0 \leq w<2^{d}$.

Lemma 18. Assuming the conditions of Theorem 15 for $d=2,3$, we can realize $f(s, t)$ with delay $d$.

Proof. First assume that $m \leq 2$. Recall that in this case, $f(s, t)$ is a symmetric binary tree. By inequality (13), we know that $W(t)+w \leq 2^{d}$. Hence, by Remark 8 , we can realize $f(s, t)$ with delay $d$ using Huffman coding. 
Now let $m \geq 3$. Requirement (11) yields

$$
1+\frac{2}{d} \stackrel{m \geq 3, \text { Rem. }}{\leq} \sum_{i=0}^{m-3} W\left(t_{i}\right)+\frac{\Lambda_{t}}{d} \stackrel{(11)}{\leq} \zeta \frac{2^{d-1}-w}{d \log _{2}(d)} \stackrel{\zeta<2}{<} \frac{2^{d}-2 w}{d \log _{2}(d)} .
$$

For $d=2$, this leads to the contradiction

$$
2=1+\frac{2}{2} \stackrel{(15)}{<} \frac{4-2 w}{2 \cdot 1} \stackrel{w \geq 0}{\leq} 2,
$$

i.e., for $d=2$, we always have $m \leq 2$ and have already proven the required statement.

Similarly, if $d=3$, we obtain

$$
\frac{5}{3}=1+\frac{2}{3} \stackrel{(15)}{<} \frac{8-2 w}{3 \cdot \log _{2}(3)}<\left\{\begin{array}{ll}
2 & w=0 \\
\frac{4}{3} & w \geq 1
\end{array} .\right.
$$

In the case that $w \geq 1$, this is a contradiction; and for $w=0$, the only remaining case is $m=3$ with $t_{0}=t_{1}=t_{2}=0$ for which $f(s, t)=t_{0} \wedge\left(t_{1} \vee t_{2}\right)$ can obviously be constructed with delay $2<3$.

Theorem 19. Assume inductively that for some $d \geq 3$ and all $0 \leq w<2^{d-1}$, Theorem 15 holds. Then, for inputs $s$ and $t$ with $w:=W(s)$ such that $0 \leq w<2^{d}$ and

$$
W(t) \leq \zeta \frac{2^{d}-w}{(d+1) \log _{2}(d+1)}+\frac{d}{d+1} \Lambda_{t},
$$

we can realize $f(s, t)$ with delay $(d+1)$.

As a sub-calculation for the proof of this theorem, we need the following lemma.

Lemma 20. In the situation of Theorem 19, we have

$$
\begin{aligned}
& \zeta \frac{2^{d-1}}{d \log _{2}(d)}+\frac{d-1}{d} \Lambda_{t}-\zeta \frac{2^{d}-w}{(d+1) \log _{2}(d+1)}-\frac{d}{d+1} \Lambda_{t} \\
\geq & \zeta \frac{2^{d-1} \log _{2}(d+1)-\left(2^{d}-w\right) \log _{2}(d)}{d \log _{2}(d) \log _{2}(d+1)} .
\end{aligned}
$$

Proof. Using the bound on $\Lambda_{t}$ implied by inequality (12), we calculate

$$
\begin{aligned}
& \zeta \frac{2^{d-1}}{d \log _{2}(d)}+\frac{d-1}{d} \Lambda_{t}-\zeta \frac{2^{d}-w}{(d+1) \log _{2}(d+1)}-\frac{d}{d+1} \Lambda_{t} \\
= & \zeta \frac{2^{d-1}(d+1) \log _{2}(d+1)-\left(2^{d}-w\right) d \log _{2}(d)}{d(d+1) \log _{2}(d) \log _{2}(d+1)}-\frac{1}{d(d+1)} \Lambda_{t} \\
\stackrel{(12)}{\geq} & \zeta \frac{2^{d-1}(d+1) \log _{2}(d+1)-\left(2^{d}-w\right) d \log _{2}(d)}{d(d+1) \log _{2}(d) \log _{2}(d+1)}-\frac{1}{d(d+1)} \zeta \frac{2^{d}-w}{\log _{2}(d+1)} \\
= & \zeta \frac{2^{d-1} \log _{2}(d+1)-\left(2^{d}-w\right) \log _{2}(d)}{d \log _{2}(d) \log _{2}(d+1)} .
\end{aligned}
$$

This is the only ingredient needed to prove Theorem 19 for the case that $2^{d-1} \leq w<2^{d}$. 
Lemma 21. Theorem 19 holds for all $w$ satisfying $2^{d-1} \leq w<2^{d}$.

Proof. The symmetric split (3) yields the realization

$$
f(s, t)=\left(s_{0} \wedge \ldots \wedge s_{n-1}\right) \wedge g(t) .
$$

Since $w<2^{d}$, Remark 8 allows the construction of a symmetric tree on inputs $s$ with delay $d$. In order to show that $f((), t)=g(t)$ can be realized with delay $d$, by induction hypothesis, it suffices to show the second inequality in

$$
W(t) \stackrel{(16)}{\leq} \zeta \frac{2^{d}-w}{(d+1) \log _{2}(d+1)}+\frac{d}{d+1} \Lambda_{t} \leq \zeta \frac{2^{d-1}}{d \log _{2}(d)}+\frac{d-1}{d} \Lambda_{t} .
$$

Subtracting the left-hand side from the right-hand side, we prove this via

$$
\begin{array}{ll} 
& \zeta \frac{2^{d-1}}{d \log _{2}(d)}+\frac{d-1}{d} \Lambda_{t}-\zeta \frac{2^{d}-w}{(d+1) \log _{2}(d+1)}-\frac{d}{d+1} \Lambda_{t} \\
\stackrel{\text { Lem. } 20}{\geq} & \zeta \frac{2^{d-1} \log _{2}(d+1)-\left(2^{d}-w\right) \log _{2}(d)}{d \log _{2}(d) \log _{2}(d+1)} \\
w^{w 2^{d-1}} & \zeta \frac{2^{d-1}\left(\log _{2}(d+1)-\log _{2}(d)\right)}{d \log _{2}(d) \log _{2}(d+1)} \\
\geq & 0 .
\end{array}
$$

Hence, applying the symmetric split (17) yields a realization for $f(s, t)$ with delay $d+1$.

In the case $0 \leq w<2^{d-1}$, we need a bound on the logarithm of consecutive integers.

Remark 22. For $d \geq 3$, we have $d \geq \ln (2)(d+1)$ and thus

$$
\log _{2}(d+1)-\log _{2}(d)=\frac{\ln (d+1)-\ln (d)}{\ln (2)}=\int_{d}^{d+1} \frac{1}{\ln (2) x} d x \geq \frac{1}{\ln (2)(d+1)} \geq \frac{1}{d}
$$

Now we will prove Theorem 19 for the case that $0 \leq w<2^{d-1}$.

Lemma 23. Theorem 19 holds for each $w$ satisfying $0 \leq w<2^{d-1}$.

Proof. We prove this lemma via a case distinction. In Case 2, we will consider a prefix $t^{\prime}$ of the inputs $t$ with weight at most $\zeta \frac{2^{d-1}-w}{d \log _{2}(d)}$ in order to proceed similarly as indicated in Remark 13 . If the weight of $t_{0}$ is already larger than this, such a prefix does not exist. We deal with this situation in Case 1.

Case 1: Assume that

$$
W\left(t_{0}\right)>\zeta \frac{2^{d-1}-w}{d \log _{2}(d)}
$$

The symmetric split (4) yields

$$
f(s, t)=\left(s_{0} \wedge \ldots \wedge s_{n-1} \wedge t_{0}\right) \wedge g^{*}\left(\left(t_{1}, t_{2}, \ldots, t_{m-1}\right)\right) .
$$

Due to inequality (13) and $d+1 \geq 4>2^{\zeta}$, we have $W\left(t_{0}\right)+w \leq W(t)+w \leq 2^{d}$. Hence, by Remark 8, we can realize $s_{0} \wedge \ldots \wedge s_{n-1} \wedge t_{0}$ as a binary tree with delay $d$. Thus, we will check 
inductively that $f^{*}\left((),\left(t_{1}, t_{2}, \ldots, t_{m-1}\right)\right)=g^{*}\left(\left(t_{1}, t_{2}, \ldots, t_{m-1}\right)\right)$ can be realized with delay $d$. Note that requirement (16) and condition (19) imply

$$
W\left(\left(t_{1}, t_{2}, \ldots, t_{m-1}\right)\right)<\zeta \frac{2^{d}-w}{(d+1) \log _{2}(d+1)}+\frac{d}{d+1} \Lambda_{t}-\zeta \frac{2^{d-1}-w}{d \log _{2}(d)}
$$

which we claim to be at most $\zeta \frac{2^{d-1}}{d \log _{2}(d)}+\frac{d-1}{d} \Lambda_{t}$. This can be shown by

$$
\begin{array}{ll} 
& \zeta \frac{2^{d-1}}{d \log _{2}(d)}+\frac{d-1}{d} \Lambda_{t}-\zeta \frac{2^{d}-w}{(d+1) \log _{2}(d+1)}-\frac{d}{d+1} \Lambda_{t}+\zeta \frac{2^{d-1}-w}{d \log _{2}(d)} \\
\stackrel{\text { Lem. } 20}{\geq} & \zeta \frac{2^{d-1} \log _{2}(d+1)-\left(2^{d}-w\right) \log _{2}(d)}{d \log _{2}(d) \log _{2}(d+1)}+\zeta \frac{2^{d-1}-w}{d \log _{2}(d)} \\
= & \zeta \frac{\left(2^{d}-w\right)\left(\log _{2}(d+1)-\log _{2}(d)\right)}{d \log _{2}(d) \log _{2}(d+1)} \\
& \\
w<2^{d} & 0 .
\end{array}
$$

Thus, realization (20) yields a delay of $d+1$ for $f(s, t)$, which proves the lemma for the case that $W\left(t_{0}\right)>\zeta \frac{2^{d-1}-w}{d \log _{2}(d)}$.

Case 2: Assume that $W\left(t_{0}\right) \leq \zeta \frac{2^{d-1}-w}{d \log _{2}(d)}$.

Therefore, we can consider a maximum odd-length prefix $t^{\prime}=\left(t_{0}, t_{1}, \ldots, t_{2 k}\right)$ of $t$ with $0 \leq$ $k \leq \frac{m-1}{2}$ such that

$$
W\left(t^{\prime}\right) \leq \zeta \frac{2^{d-1}-w}{d \log _{2}(d)}
$$

We define $t^{\prime \prime}:=\left(t_{2 k+1}, \ldots, t_{m-1}\right)$.

If $t^{\prime \prime}$ is empty, there is nothing to show since, by induction hypothesis, we can construct $f(s, t)=f\left(s, t^{\prime}\right)$ with a delay of $d<d+1$ due to $w<2^{d-1}$. Otherwise, we will realize $f(s, t)$ with delay $d+1$ using the alternating split (5) for some prefix $t^{*}$ of $t$ to be determined, i.e.,

$$
f(s, t)=f\left(s, t^{*}\right) \wedge f^{*}\left(\widehat{t^{*}}, t^{* *}\right),
$$

where $t^{*}=\left(t_{0}, t_{1}, \ldots, t_{2 l}\right)$ for some $0 \leq l<\frac{m-1}{2}$ and $t^{* *}:=\left(t_{2 l+1}, \ldots, t_{m-1}\right)$. Our main argument, which is presented in Case 2 (ii), requires that $\left\{t_{2 k+1}, t_{2 k+2}\right\} \cap\left\{t_{m-2}, t_{m-1}\right\}=\emptyset$, i.e., that $t^{\prime \prime}$ has at least 4 elements. Thus, in Case 2 (i), we treat the $t^{\prime \prime}$ with at most 2 elements, and at the beginning of Case 2 (ii) those with exactly 3 elements.

Case 2 (i): Assume that $t^{\prime \prime}$ consists of at most 2 elements.

We set $t^{*}:=t^{\prime}$, thus $t^{* *}=t^{\prime \prime}$. By induction hypothesis and due to $w<2^{d-1}$, inequality $(21)$ allows realizing $f\left(s, t^{\prime}\right)$ with delay $d$. Since $t^{\prime \prime}$ has at most 2 elements, by Remark 8 , we can realize $f^{*}\left(\widehat{t^{\prime}}, t^{\prime \prime}\right)$ as a binary tree with delay $d$ since $W\left(\widehat{t^{\prime}}\right)+W\left(t^{\prime \prime}\right) \leq W(t)$, which is at most $2^{d}$ due to inequality (13) and $d+1 \geq 4>2^{\zeta}$.

Case 2 (ii): Assume that $t^{\prime \prime}$ contains at least 3 elements.

Set $\tilde{t}:=\left(t_{0}, \ldots, t_{2 k+2}\right)$. We need to find an appropriate prefix $t^{*}$ of $t$ for realization (22) such that both $f\left(s, t^{*}\right)$ and $f^{*}\left(\widehat{t^{*}}, t^{* *}\right)$ can be realized with delay $d$ by induction hypothesis. We choose $t^{*}$ depending on the weight of $\tilde{t}$ :

a. If $W(\tilde{t}) \leq \zeta \frac{2^{d-1}-w}{d \log _{2}(d)}+\frac{d-1}{d} \Lambda_{\tilde{t}}$, we set $t^{*}:=\tilde{t}$.

b. If $W(\tilde{t})>\zeta \frac{2^{d-1}-w}{d \log _{2}(d)}+\frac{d-1}{d} \Lambda_{\tilde{t}}$, we set $t^{*}:=t^{\prime}$. Note that in this case, we in particular have

$$
W\left(t^{*}\right)=W\left(t^{\prime}\right)=W(\tilde{t})-\Lambda_{\tilde{t}}>\zeta \frac{2^{d-1}-w}{d \log _{2}(d)}+\frac{d-1}{d} \Lambda_{\tilde{t}}-\Lambda_{\tilde{t}}=\zeta \frac{2^{d-1}-w}{d \log _{2}(d)}-\frac{1}{d} \Lambda_{\tilde{t}} .
$$




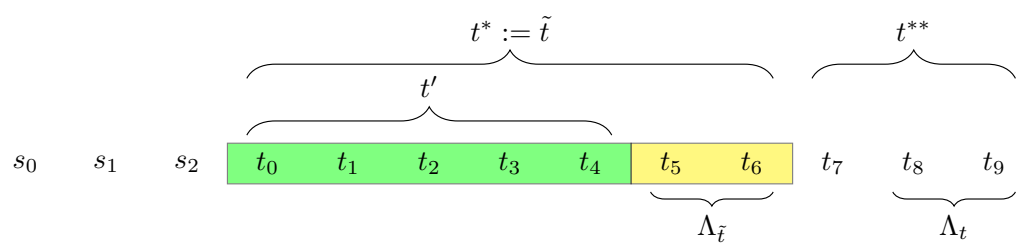

(a) In the case that $W(\tilde{t}) \leq \frac{2^{d-1}-w}{d \log _{2}(d)}$, we set $t^{*}:=\tilde{t}$.

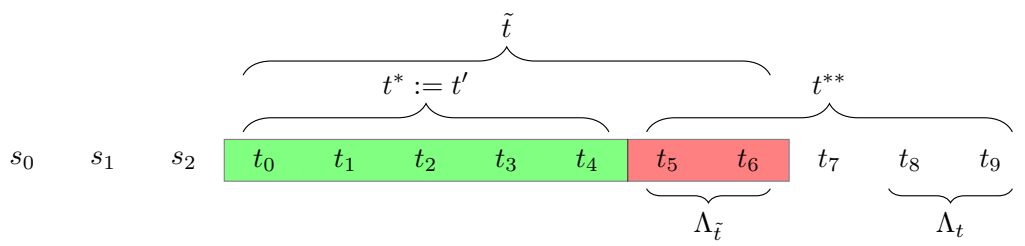

(b) In the case that $W(\tilde{t})>\frac{2^{d-1}-w}{d \log _{2}(d)}$, we set $t^{*}:=t^{\prime}$.

Figure 3: Illustration of the choice of $t^{*}$.

Figure 3 visualizes the case distinction. In either case, the weight of $t^{*}$ will be of the form

$$
W\left(t^{*}\right)=\zeta \frac{2^{d-1}-w}{d \log _{2}(d)}+\delta \text { with }-\frac{1}{d} \Lambda_{\tilde{t}} \leq \delta \leq \frac{d-1}{d} \Lambda_{\tilde{t}} .
$$

The upper bound on $\delta$ allows us to realize $f\left(s, t^{*}\right)$ with delay $d$ by induction hypothesis since $w<2^{d-1}$. It remains to show that $f^{*}\left(\widehat{t^{*}}, t^{* *}\right)$ can be realized with delay $d$.

The case that $t^{\prime \prime}$ contains exactly 3 elements still needs to be treated separately. Here, case (a) is easy since we have $t^{* *}=\left(t_{m-1}\right)$, hence $f^{*}\left(\widehat{t^{*}}, t^{* *}\right)$ is a binary tree which can be realized with delay $d$ by Huffman coding since $W(t) \leq 2^{d}$ due to inequality (13). In Case (b), we show that the realization

$$
f^{*}\left(\widehat{t^{\prime}},\left(t_{2 k+1}, t_{2 k+2}, t_{2 k+3}\right)\right)=\left(\widehat{t^{\prime}} \vee t_{2 k+1}\right) \vee\left(t_{2 k+2} \wedge t_{2 k+1}\right)
$$

yields delay $d$ : The binary tree $\widehat{t^{\prime}} \vee t_{2 k+1}$ can be realized with delay $d-1$ using Remark 8 since

$$
W\left(\widehat{t^{\prime}}\right)+W\left(t_{2 k+1}\right) \stackrel{(11)}{\leq} \zeta \frac{2^{d}-w}{(d+1) \log _{2}(d+1)} \stackrel{\zeta<2, w \geq 0}{<} \frac{2^{d+1}}{(d+1) \log _{2}(d+1)} \stackrel{d \geq 2}{\leq} 2^{d-1} .
$$

It remains to show $\Lambda_{t} \leq 2^{d-1}$, so assume the contrary. W.l.o.g., since $f(s, t)$ is logically symmetric in $t_{2 k+2}$ and $t_{2 k+3}$, we may assume $W\left(t_{2 k+3}\right)=\max \left\{W\left(t_{2 k+3}\right), W\left(t_{2 k+2}\right)\right\}$. Due to inequality (13) and $m \geq 4$, we have $2^{d-1}<\Lambda_{t}<2^{d}$. This implies $W\left(t_{2 k+3}\right)=2^{d-1}$ and $W\left(t_{2 k+2}\right) \leq 2^{d-2}$. By combining

$W(t)-\Lambda_{t} \stackrel{(16)}{\leq} \zeta \frac{2^{d}-w}{(d+1) \log _{2}(d+1)}-\frac{\Lambda_{t}}{d+1}=\zeta \frac{2^{d}-w}{(d+1) \log _{2}(d+1)}-\frac{W\left(t_{2 k+2}\right)+W\left(t_{2 k+3}\right)}{d+1}$

and

$$
\begin{aligned}
W(t)-\Lambda_{t}=W(\tilde{t})-W\left(t_{2 k+2}\right) & \stackrel{\text { case (b) }}{>} \zeta \frac{2^{d-1}-w}{d \log _{2} d}+\frac{d-1}{d} \Lambda_{\tilde{t}}-W\left(t_{2 k+2}\right) \\
& \stackrel{(7)}{\geq} \zeta \frac{2^{d-1}-w}{d \log _{2} d}+\frac{d-1}{d}-\frac{W\left(t_{2 k+2}\right)}{d},
\end{aligned}
$$


we obtain

$$
\begin{aligned}
& 0<\quad \zeta \frac{2^{d}-w}{(d+1) \log _{2}(d+1)}-\zeta \frac{2^{d-1}-w}{d \log _{2} d}-\frac{W\left(t_{2 k+2}\right)+W\left(t_{2 k+3}\right)}{d+1}-\frac{d-1}{d}+\frac{W\left(t_{2 k+2}\right)}{d} \\
& \underset{w<2^{d-1}}{<} \quad \zeta \frac{2^{d-1}}{(d+1) \log _{2}(d+1)}+\frac{W\left(t_{2 k+2}\right)-d W\left(t_{2 k+3}\right)-d^{2}+1}{d(d+1)} \\
& \begin{array}{l}
W\left(t_{2 k+2}\right) \leq 2^{d-2}, \\
W\left(t_{2 k+3}\right)=2^{d-1} \\
\leq
\end{array} \quad \frac{2^{d-1}}{(d+1) \log _{2}(d+1)}+\frac{2^{d-2}-d 2^{d-1}-d^{2}+1}{d(d+1)} \\
& \stackrel{\zeta<2}{<} \frac{d 2^{d}+\log _{2}(d+1)\left(2^{d-2}-d 2^{d-1}-d^{2}+1\right)}{d(d+1) \log _{2}(d+1)} \\
& <\quad 0 \text {, }
\end{aligned}
$$

where the last step can be verfied by hand for $d=3$, and for $d \geq 4$ is implied by

$d 2^{d}+\log _{2}(d+1)\left(2^{d-2}-d 2^{d-1}\right)=2^{d-2}\left(4 d+\log _{2}(d+1)(1-2 d)\right) \stackrel{d \geq 4}{\leq} 2^{d-2}\left(4 d-7 \log _{2}(5)\right) \stackrel{d \geq 4}{\gtrless} 0$.

This is a contradiction, concluding the case that $t^{\prime \prime}$ has exactly 3 elements.

Now we may assume that $t^{\prime \prime}$ contains at least 4 elements. In particular, the first two elements $t_{2 k+1}$ and $t_{2 k+2}$ of $t^{\prime \prime}$ and the last two elements $t_{m-2}$ and $t_{m-1}$ of $t$ are disjoint sets. Note that since $t^{*}$ does not contain any of the last two elements of $t$, we have

$$
W\left(\widehat{t^{*}}\right) \stackrel{(11)}{\leq} \zeta \frac{2^{d}-w}{(d+1) \log _{2}(d+1)} \stackrel{w \geq 0}{\leq} \zeta \frac{2^{d}}{(d+1) \log _{2}(d+1)} \stackrel{\zeta<2}{<} 2^{d-1}
$$

for $d \geq 2$ and thus, by induction hypothesis, it suffices to prove that

$$
W\left(t^{* *}\right) \leq \zeta \frac{2^{d-1}-W\left(\widehat{t^{*}}\right)}{d \log _{2}(d)}+\frac{d-1}{d} \Lambda_{t^{* *}}
$$

Due to requirement (16), we have

$$
W\left(t^{* *}\right)=W(t)-W\left(t^{*}\right) \leq \zeta \frac{2^{d}-w}{(d+1) \log _{2}(d+1)}+\frac{d}{d+1} \Lambda_{t}-W\left(t^{*}\right) .
$$

Since $W\left(\widehat{t^{*}}\right) \leq W\left(t^{*}\right)$ and $\Lambda_{t^{* *}}=\Lambda_{t}$, inequality (24) is thus implied if we prove the following claim.

Claim: We have $\zeta \frac{2^{d-1}-W\left(t^{*}\right)}{d \log _{2}(d)}+\frac{d-1}{d} \Lambda_{t}-\zeta \frac{2^{d}-w}{(d+1) \log _{2}(d+1)}-\frac{d}{d+1} \Lambda_{t}+W\left(t^{*}\right) \geq 0$.

Proof of the claim: We first only bound the summands depending on $W\left(t^{*}\right)$ or $\Lambda_{t}$.

$$
\begin{array}{ll} 
& -\zeta \frac{W\left(t^{*}\right)}{d \log _{2}(d)}+\frac{d-1}{d} \Lambda_{t}-\frac{d}{d+1} \Lambda_{t}+W\left(t^{*}\right) \\
= & W\left(t^{*}\right)\left(1-\frac{\zeta}{d \log _{2}(d)}\right)-\frac{1}{d(d+1)} \Lambda_{t} \\
\stackrel{(23)}{\geq} & \left(\zeta \frac{2^{d-1}-w}{d \log _{2}(d)}-\frac{1}{d} \Lambda_{\tilde{t}}\right) \frac{d \log _{2}(d)-\zeta}{d \log _{2}(d)}-\frac{1}{d(d+1)} \Lambda_{t} \\
= & \zeta \frac{\left(2^{d-1}-w\right)\left(d \log _{2}(d)-\zeta\right)}{d^{2} \log _{2}^{2}(d)}-\frac{d \log _{2}(d)-\zeta}{d^{2} \log _{2}(d)} \Lambda_{\tilde{t}}-\frac{1}{d(d+1)} \Lambda_{t}
\end{array}
$$




$$
\begin{aligned}
= & \zeta \frac{\left(2^{d-1}-w\right)\left(d \log _{2}(d)-\zeta\right)}{d^{2} \log _{2}^{2}(d)}-\frac{1}{d}\left(\Lambda_{\tilde{t}}+\frac{\Lambda_{t}}{d+1}\right)+\frac{\zeta \Lambda_{\tilde{t}}}{d^{2} \log _{2}(d)} \\
\stackrel{(11)}{\geq} & \zeta \frac{\left(2^{d-1}-w\right)\left(d \log _{2}(d)-\zeta\right)}{d^{2} \log _{2}^{2}(d)}-\frac{1}{d}\left(\zeta \frac{2^{d}-w}{(d+1) \log _{2}(d+1)}-W\left(t^{\prime}\right)\right)+\frac{\zeta \Lambda_{\tilde{t}}}{d^{2} \log _{2}(d)} \\
\stackrel{\text { Rem. } 7}{\geq} & \zeta \frac{\left(2^{d-1}-w\right)\left(d \log _{2}(d)-\zeta\right)}{d^{2} \log _{2}^{2}(d)}-\zeta \frac{2^{d}-w}{d(d+1) \log _{2}(d+1)}+\frac{1}{d}+\frac{2 \zeta}{d^{2} \log _{2}(d)}
\end{aligned}
$$

Note that in the last two steps, we used that $t_{2 k+1}, t_{2 k+2}, t_{m-2}, t_{m-1}$ are four different inputs which are not contained in $t^{\prime}$ and that $t^{\prime}$ is not empty. Based on inequality (25), the left-hand side of the claim can be bounded from below by

$$
\begin{aligned}
& \zeta \frac{2^{d-1}-W\left(t^{*}\right)}{d \log _{2}(d)}+\frac{d-1}{d} \Lambda_{t}-\zeta \frac{2^{d}-w}{(d+1) \log _{2}(d+1)}-\frac{d}{d+1} \Lambda_{t}+W\left(t^{*}\right) \\
\stackrel{(25)}{\geq} & \zeta\left(\frac{\left(2^{d-1}-w\right)\left(d \log _{2}(d)-\zeta\right)}{d^{2} \log _{2}^{2}(d)}-\frac{2^{d}-w}{d(d+1) \log _{2}(d+1)}+\frac{1}{d}+\frac{2 \zeta}{d^{2} \log _{2}(d)}+\frac{2^{d-1}}{d \log _{2}(d)}\right. \\
& \left.\quad-\frac{2^{d}-w}{(d+1) \log _{2}(d+1)}\right) \\
= & \zeta\left(\frac{2^{d-1}-w+2^{d-1}}{d \log _{2}(d)}-\zeta \frac{2^{d-1}-w}{d^{2} \log _{2}^{2}(d)}-\frac{\left(2^{d}-w\right)(d+1)}{d(d+1) \log _{2}(d+1)}+\frac{1}{d}+\frac{2 \zeta}{d^{2} \log _{2}(d)}\right) \\
= & \zeta\left(\frac{2^{d}-w}{d \log _{2}(d)}-\zeta \frac{2^{d-1}-w}{d^{2} \log _{2}^{2}(d)}-\frac{2^{d}-w}{d \log _{2}(d+1)}+\frac{1}{d}+\frac{2 \zeta}{d^{2} \log _{2}(d)}\right) \\
= & \frac{\zeta}{d^{2} \log _{2}^{2}(d) \log _{2}(d+1)}\left(\log _{2}(d+1)\left(\left(2^{d}-w\right) d \log _{2}(d)-\zeta\left(2^{d-1}-w\right)\right)\right. \\
& \left.-\left(2^{d}-w\right) d \log _{2}^{2}(d)+\left(2+\frac{1}{\zeta} d \log _{2}(d)\right) \log _{2}(d) \log _{2}(d+1)\right),
\end{aligned}
$$

which is required to be non-negative. After multiplying with the denominator and dividing by $\zeta$, we apply the bound $\log _{2}(d+1) \geq \log _{2}(d)+\frac{1}{d}$ stated in Remark 22, and thus can prove the claim if we show that

$$
\begin{array}{ll} 
& \log _{2}(d+1)\left(\left(2^{d}-w\right) d \log _{2}(d)-\zeta\left(2^{d-1}-w\right)\right) \\
& -\left(2^{d}-w\right) d \log _{2}^{2}(d)+\left(2+\frac{1}{\zeta} d \log _{2}(d)\right) \log _{2}(d) \log _{2}(d+1) \\
\stackrel{\zeta<2,(18)}{\geq} & \left(2^{d}-w\right) \log _{2}(d)-\left(\log _{2}(d)+\frac{1}{d}\right) \zeta\left(2^{d-1}-w\right)+\left(2+\frac{1}{\zeta} d \log _{2}(d)\right) \log _{2}(d) \log _{2}(d+1) \\
\stackrel{\zeta \geq 1, w \geq 0}{\geq} & 2^{d} \log _{2}(d)-\left(\log _{2}(d)+\frac{1}{d}\right) \zeta 2^{d-1}+\left(2+\frac{1}{\zeta} d \log _{2}(d)\right) \log _{2}(d) \log _{2}(d+1)
\end{array}
$$

is at least 0 . Note that for $d \geq 7$, this is already implied by

$$
2^{d} \log _{2}(d)-\left(\log _{2}(d)+\frac{1}{d}\right) \zeta 2^{d-1} \stackrel{\zeta=1.9}{=} 2^{d-1}\left(0.1 \log _{2}(d)-\frac{1.9}{d}\right) \stackrel{d \geq 7}{\geq} 0 .
$$


For $3 \leq d \leq 6$, we have

$$
\begin{aligned}
& 2^{d} \log _{2}(d)-\left(\log _{2}(d)+\frac{1}{d}\right) \zeta 2^{d-1}+\left(2+\frac{1}{\zeta} d \log _{2}(d)\right) \log _{2}(d) \log _{2}(d+1) \\
\stackrel{\zeta=1.9}{=} & \log _{2}(d)\left(0.1 \cdot 2^{d-1}+\left(2+\frac{1}{1.9} d \log _{2}(d)\right) \log _{2}(d+1)\right)-\frac{1.9}{d} 2^{d-1} \\
\stackrel{d \geq 3}{\geq} & \log _{2}(3)\left(0.1 \cdot 2^{2}+\left(2+\frac{1}{1.9} 3 \log _{2}(3)\right) 2\right)-\frac{1.9}{d} 2^{d-1} \\
> & 14-\frac{1.9}{d} 2^{d-1} \\
\stackrel{d \leq 6}{\geq} & 14-\frac{1.9}{6} 32 \\
> & 0 .
\end{aligned}
$$

This proves the claim. Note that this is the only place where we used the definition $\zeta=1.9$.

Thus, by induction hypothesis, we can find a realization with delay $d$ for $f\left(\widehat{t^{*}}, t^{* *}\right)$. Split $(22)$ hence also provides a realization with delay $d+1$ for $f(s, t)$ if $t^{\prime \prime}$ contains at least 4 elements. This concludes the proof.

Proof of Theorem 19. Lemma 23 proves the theorem in the case that $0 \leq w<2^{d-1}$, while Lemma 21 proves it for the remaining case that $2^{d-1} \leq w<2^{d}$.

Finally, we can prove Theorem 15.

Proof of Theorem 15. We prove the theorem by induction on $d$. For $d \leq 3$, Lemma 18 provides a realization of $f(s, t)$ with delay $d$. Now we can assume that the theorem holds for some $d \geq 3$, and prove the inductive step via Theorem 19.

\section{Constructing Fast Circuits}

Based on Theorem 11, we could now show that there is a circuit realizing the AND-Or path $t_{0} \wedge\left(t_{1} \vee\left(t_{2} \wedge\left(\ldots t_{m-1}\right) \ldots\right)\right.$ with delay at most $\log _{2} W+\log _{2} \log _{2} W+\log _{2} \log _{2} \log _{2} W+5$. Instead, we will prove a stronger result: By modifying the instance, we can diminish the dependency on $W$. The modification is based on the observation that we can round up small arrival times to the same value without losing too much for the maximum delay. Moreover, shifting all arrival times by some number does not change the problem. Both modifications allow us to reduce the problem to instances with a total arrival time weight of at most $2 m$.

Theorem 24. Let $m \in \mathbb{N}$ with $m \geq 3$, Boolean variables $t_{0}, \ldots, t_{m-1}$ and arrival times a: $\left\{t_{0}, \ldots, t_{m-1}\right\} \rightarrow \mathbb{N}$ be given, and define $W:=\sum_{i=0}^{m-1} 2^{a\left(t_{i}\right)}$. There is circuit realizing the ANDOR path $t_{0} \wedge\left(t_{1} \vee\left(t_{2} \wedge\left(\ldots t_{m-1}\right) \ldots\right)\right.$ with delay at most

$$
\log _{2} W+\log _{2} \log _{2} m+\log _{2} \log _{2} \log _{2} m+4.3 .
$$

Proof. We compute new arrival times $\tilde{a}:\left\{t_{0}, \ldots, t_{m-1}\right\} \rightarrow \mathbb{N}$ by setting

$$
\tilde{a}\left(t_{i}\right):=\max \left\{0, a\left(t_{i}\right)-\left\lceil\left(\log _{2} W-\log _{2} m\right)\right\rceil\right\}
$$


for all $i \in\{0, \ldots, m-1\}$. We define $\widetilde{W}:=\sum_{i=0}^{m-1} 2^{\tilde{a}\left(t_{i}\right)}$ and partition the input indices into $I_{1}:=\left\{i \in\{0, \ldots, m-1\} \mid \tilde{a}\left(t_{i}\right)=0\right\}$ and $I_{2}:=\{0, \ldots, m-1\} \backslash I_{1}$. Then, we have

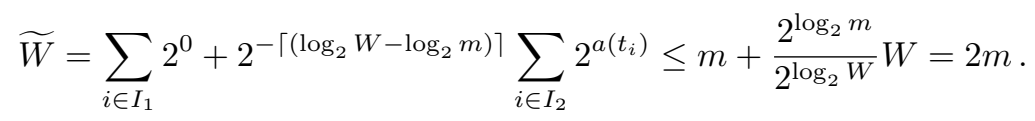

Define $\tilde{d}:=\left\lfloor\log _{2} m+\log _{2} \log _{2} m+\log _{2} \log _{2} \log _{2} m+3.3\right\rfloor$.

Claim: There is a circuit $C$ realizing the AND-OR path $t_{0} \wedge\left(t_{1} \vee\left(t_{2} \wedge\left(\ldots t_{m-1}\right) \ldots\right)\right.$ with arrival times $\tilde{a}$ with delay at most $\tilde{d}$.

Proof of the claim: Let $M:=500$. If $m<M$, we have $1.441 \log _{2} \widetilde{W}+2.674 \leq 1.441 \log _{2}(2 m)+$ $2.674=1.441 \log _{2} m+4.115 \leq \log _{2} m+\log _{2} \log _{2} m+\log _{2} \log _{2} \log _{2} m+3.3$. Since the ANDOr path optimization method by Held and Spirkl [7] computes a circuit with delay at most $\left\lfloor 1.441 \log _{2} \widetilde{W}+2.674\right\rfloor$, this proves the claim for $m<M$.

Hence assume $m \geq M$. For proving the claim, by Theorem 11, it is sufficient to show

$$
2 m \leq \zeta \frac{2^{\tilde{d}-1}}{\tilde{d} \log _{2} \tilde{d}} .
$$

Note that the mapping $x \mapsto \frac{2^{x-1}}{x \log _{2} x}$ is strictly increasing for $x \geq 2$. Moreover, we have $\tilde{d} \geq$ $\log _{2} m+\log _{2} \log _{2} m+\log _{2} \log _{2} \log _{2} m+2.3$. Since

$$
\log _{2} m+\log _{2} \log _{2} m+\log _{2} \log _{2} \log _{2} m+2.3 \leq 1.8 \log _{2} m
$$

for $m \geq M$, equation (26) is hence valid if

$$
2 m \leq \zeta \frac{\frac{1}{2} \cdot m \cdot \log _{2} m \cdot \log _{2} \log _{2} m \cdot 2^{2.3}}{1.8 \cdot \log _{2} m \cdot \log _{2}\left(1.8 \cdot \log _{2} m\right)} .
$$

This is equivalent to

$$
1.8 \cdot \log _{2}\left(1.8 \cdot \log _{2} m\right) \leq \zeta 2^{0.3} \cdot \log _{2} \log _{2} m,
$$

which is true for $m \geq M$ since $\zeta=1.9$. This proves the claim.

Since we have $a\left(t_{i}\right) \leq \tilde{a}\left(t_{i}\right)+\left\lceil\left(\log _{2} W-\log _{2} m\right)\right\rceil$ for all $i \in\{0, \ldots, m-1\}$, the circuit $C$ has, for the initial arrival times $a:\left\{t_{0}, \ldots, m-1\right\} \rightarrow \mathbb{N}$, a delay of at most

$$
\begin{aligned}
& \left\lfloor\log _{2} m+\log _{2} \log _{2} m+\log _{2} \log _{2} \log _{2} m+3.3\right\rfloor+\left\lceil\left(\log _{2} W-\log _{2} m\right)\right\rceil \\
\leq & \log _{2} W+\log _{2} \log _{2} m+\log _{2} \log _{2} \log _{2} m+4.3 .
\end{aligned}
$$

Remark 25. In the proof, we apply the algorithm from Held and Spirkl [7] for small instances. Without this trick, we would obtain a delay bound of $\log _{2} W+\log _{2} \log _{2} m+\log _{2} \log _{2} \log _{2} m+7$. Moreover, for sufficiently large values of $m$, the delay bound in the previous theorem can be improved slightly to $\log _{2} W+\log _{2} \log _{2} m+\log _{2} \log _{2} \log _{2} m+4+\varepsilon$ for any constant $\varepsilon>0$ : Note that the factor 1.8 in inequality (27) can be decreased to a value arbitrarily close to 1 if $m$ is sufficiently large. Thus, also the factor $\zeta 2^{0.3}$ in inequality (28) becomes arbitrarily close to 1 for large values of $m$. This leads to the stated delay bound.

The following theorem shows that the circuit described in Theorem 24 does not only exist, but can also be computed efficiently.

Theorem 26. There is an algorithm that computes the circuit in Theorem 24 for given $m \geq 3$ in time $\mathcal{O}\left(m^{2} \log _{2} m\right)$. 
Proof. As main subroutine, we will use Algorithm 1.

Claim: Given input variables $s=\left(s_{1}, \ldots, s_{n-1}\right)$ and $t=\left(t_{0}, \ldots, t_{m-1}\right)$ with arrival times $a$ : $\left\{t_{0}, \ldots, t_{m-1}, s_{0}, \ldots, s_{n-1}\right\} \rightarrow \mathbb{N}$, Algorithm 1 computes a Boolean circuit realizing $f(s, t)$ with delay at most $d$, where $d$ is the smallest natural number with $w:=W(s)<2^{d-1}$ and $W(t) \leq$ $\zeta \frac{2^{d-1}-w}{d \log _{2}(d)}+\frac{d-1}{d} \Lambda_{t}$. The number of computation steps of Algorithm 1 is

$$
\mathcal{O}\left(m(m+n) \log _{2}(m+n)+m \log _{2} \log _{2}\left(W^{\prime}\right)\right)
$$

where $W^{\prime}=\sum_{i=0}^{m-1} 2^{a\left(t_{i}\right)}+\sum_{i=0}^{n-1} 2^{a\left(s_{i}\right)}$.

Proof of the claim: We apply the recursive approach described in Algorithm 1 which arises from the proof of Theorem 15: In line 1, we compute the minimum $d \in \mathbb{N}$ such that $W(t) \leq$ $\zeta \frac{2^{d-1}-w}{d \log _{2}(d)}+\frac{d-1}{d} \Lambda_{t}$. We have $d \in \mathcal{O}\left(\log _{2}\left(W^{\prime}\right)\right)$, so $d$ can be computed by binary search in $\mathcal{O}\left(\log _{2} \log _{2}\left(W^{\prime}\right)\right)$ steps. Note that in line 1 , we have $w<2^{d-1}$ since otherwise, we would obtain a contradiction to $\Lambda_{t} \leq W(t)$ since

$$
W(t) \leq \zeta \frac{2^{d-1}-w}{d \log _{2}(d)}+\frac{d-1}{d} \Lambda_{t}<\frac{d-1}{d} \Lambda_{t}<\Lambda_{t} .
$$

Thus, Theorem 15 provides a circuit realizing $f(s, t)$ with delay $d$. Lemma 18 computes this realization if $d \leq 3$ (see lines 4 to 7 ). For $d>3$, Lemmata 21 (see lines 11 to 13 ) and 23 (see lines 16 to 37) construct the circuit recursively. Hence, the claimed delay bound is fulfilled by Theorem 15.

We prove the bound on the number of computation steps of Algorithm 1 by counting the number of steps needed for a single call excluding the recursive calls (i.e., lines 12, 18, 27, 35) and bounding the number of recursion steps.

Note that whenever two non-disjoint sequences of inputs are considered as alternating inputs in the algorithm, one of the sequences must be a subset of the other. Therefore, the number of alternating input sequences considered by the algorithm can be bounded by $m$. Moreover, in each of the recursive calls in lines 18, 27, 35, the number of alternating inputs decreases by 1 , and in the only other recursive call in line 12 , the number remains the same, but in this case, we recursively compute $f((), t)$, thus the number of alternating inputs will decrease in the next recursive call. Thus, the number of recursive calls in the algorithm is bounded by $2 m$.

Note that in each call of Algorithm 1, we compute at most one symmetric binary tree. Since each symmetric tree we construct has at most $m+n$ inputs, due to Remark 8 , this takes $\mathcal{O}\left((m+n) \log _{2}(m+n)\right)$ steps per tree.

If we precompute the weight for each consecutive subset of $t$, computing the prefix $t^{\prime}$ in line 22 (or finding out that no such prefix exists) requires $\mathcal{O}\left(\log _{2} m\right)$ steps using binary search.

Apart from this, there are only constantly many steps in each recursive call.

Hence, the number of steps needed for each recursive call of Algorithm 1, excluding lines 12 , 18,27 , and 35 , is at most $\mathcal{O}\left((m+n) \log _{2}(m+n)+\log _{2} \log _{2}\left(W^{\prime}\right)\right)$. Since there are at most $2 m$ recursive calls, we have at $\operatorname{most} \mathcal{O}\left(m(m+n) \log _{2}(m+n)+m \log _{2} \log _{2}\left(W^{\prime}\right)\right)$ steps in total, which finishes the proof of the claim.

Now we can prove the theorem. We follow the proof of Theorem 24, also using its notation. For $m<M$, we construct the circuit described in [7] such that nothing more is to show due to the properties collected in Table 1 . For $m \geq M$, we compute the modified instance with arrival times $\tilde{a}$ and weight $\widetilde{W}$ in linear time. Then, we call Algorithm 1 with the modified arrival times $\tilde{a}$. Since $\widetilde{W} \in \mathcal{O}(m)$, the sizes of all numbers occurring in the algorithm are polynomial in $m$. Applying the claim with $s=()$, hence $n=0$, and $W^{\prime}=\widetilde{W}$, we obtain a running time of $\mathcal{O}\left(m^{2} \log _{2}(m)\right)$. 


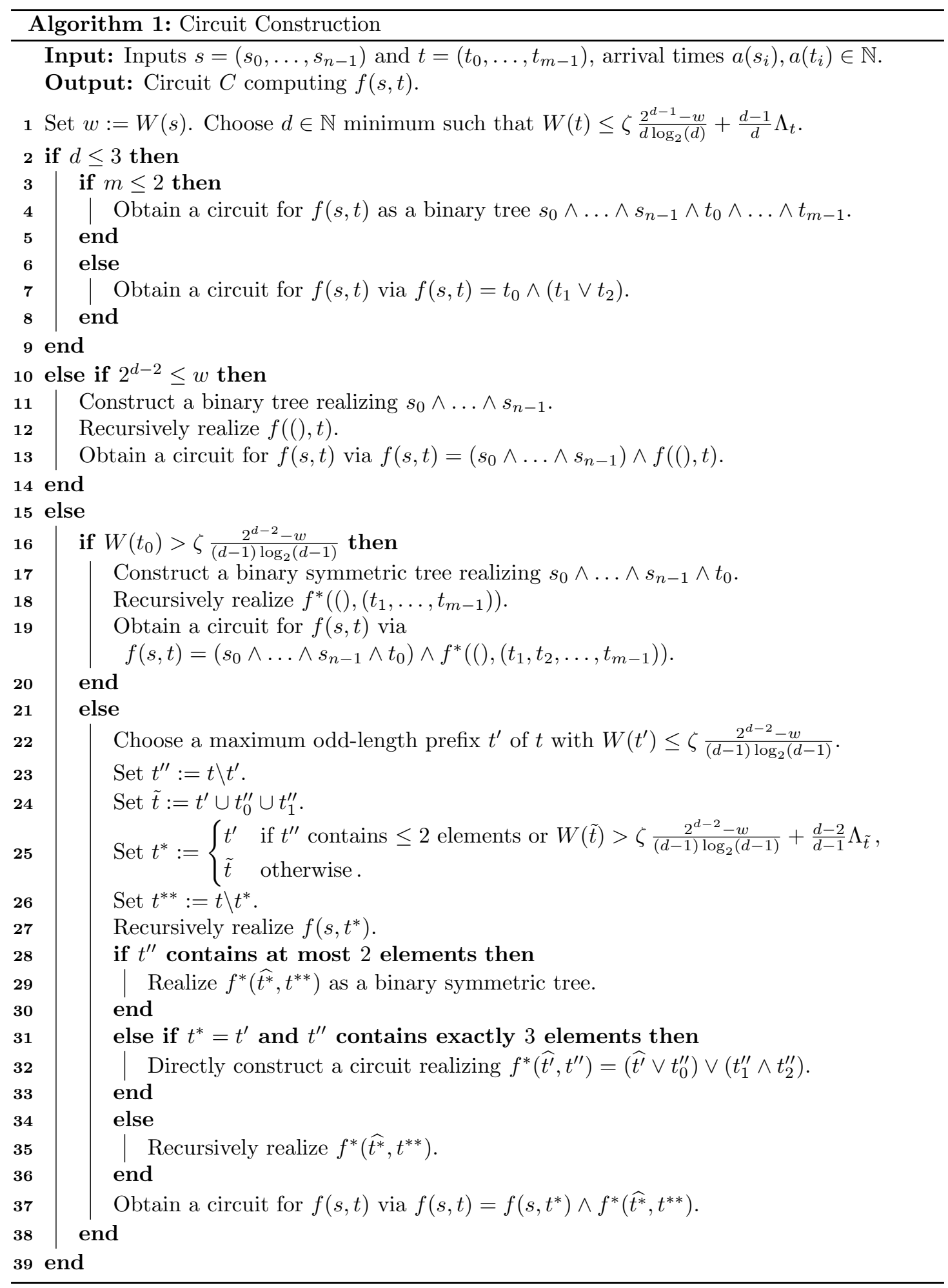


Our main objective when designing good circuits for AND-OR paths is delay. Still, there are other metrics to be regarded during circuit construction such as the size, i.e., the total number of gates used in the circuit, and maximum fanout, i.e., the maximum number of successors of any input or gate.

Theorem 27. The circuit computed in Theorem 26 has size at most

$$
m\left(\log _{2} m+\log _{2} \log _{2} m+\log _{2} \log _{2} \log _{2} m+3.3\right)
$$

and maximum fanout at most

$$
\log _{2} m+\log _{2} \log _{2} m+\log _{2} \log _{2} \log _{2} m+3.3 .
$$

Proof. In order to prove the fanout bound, we show the following claim.

Claim: In the circuit computed by Algorithm 1, each gate has fanout exactly 1, each input in $s$ has fanout exactly 1 and each input in $t$ has fanout at most $d$.

Proof of the claim: Note that each gate constructed has fanout 1 . We prove the bound on the maximum fanout of the inputs by induction on $d$.

Note that in the realizations computed by Lemma 18 which is used in lines 4 to 7 , each input has fanout 1 . In the realizations provided in lines 13 and 19, inputs of $s$ only occur in exactly one binary tree and thus have a fanout of 1 . Since we can inductively assume that $f((), t)$ and $f\left((),\left(t_{1}, \ldots, t_{m-1}\right)\right)$ fulfill the claimed fanout bound, respectively, each input in $t$ has fanout at most $d-1<d$.

In the realization in line 37 , inductively, the circuit for $f\left(s, t^{*}\right)$ computed in line 35 has fanout at most 1 for inputs in $s$ and fanout at most $d-1$ for inputs in $t^{*}$. Since inputs of $s$ do not occur in $f^{*}\left(\widehat{t^{*}}, t^{* *}\right)$, it remains to show that inputs of $t$ have fanout at most $d$ in the realization for $f(s, t)$. If $t^{\prime \prime}$ has at most 3 inputs, lines 29 and 32 show that each input of $t$ has fanout at most 1 in the realization of $f^{*}\left(\widehat{t}^{*}, t^{* *}\right)$, which proves the claimed fanout bounds. Otherwise, we realize $f^{*}\left(\widehat{t^{*}}, t^{* *}\right)$ recursively in line 35 and inductively can assume that the inputs of $\widehat{t^{*}}$ have fanout at most 1 and the inputs of $t^{* *}$ fanout at most $d-1$ in this realization. Together with the recursive fanout bounds for the realization of $f\left(s, t^{*}\right)$, this shows the claimed fanout bounds for the circuit constructed for $f(s, t)$. This proves the claim.

For the bound on the size, recall that the circuit we construct is a formula. Hence, the size is exactly $\sum_{i}$ fanout $\left(t_{i}\right)-1$. By the proven fanout bound, this is at most

$$
m\left(\log _{2} m+\log _{2} \log _{2} m+\log _{2} \log _{2} \log _{2} m+3.3\right) .
$$

Remark 28. For given $m \geq 3$, with the additional use of buffers, the circuit constructed in Theorem 26 can be transformed into a logically equivalent circuit with maximum fanout 2 , but with delay at most

$$
\log _{2} W+2 \log _{2} \log _{2} m+\log _{2} \log _{2} \log _{2} m+6
$$

and size at most $\mathcal{O}\left(m \log _{2} m \log _{2} \log _{2} m\right)$.

To see this, first note that the circuit constructed in [7] which we use for small instances already has a maximum fanout of 2 . Secondly, note that the circuit $C$ constructed in Theorem 26 has fanout larger than 1 only at the inputs. Write

$$
f:=\log _{2} m+\log _{2} \log _{2} m+\log _{2} \log _{2} \log _{2} m+3.3
$$

for the maximum possible fanout of $C$. For each input $t_{i}$, we can replace the outgoing edges of $t_{i}$ by a delay-optimum buffer tree with maximum fanout 2 for each buffer (compare Remark 8). This increases the size by at most $m(f-1)$ and, since we can assume that $m \geq 500$, the delay by at $\operatorname{most} \log _{2} f \leq \log _{2} \log _{2} m+1$. This yields the stated properties of the transformed circuit. 


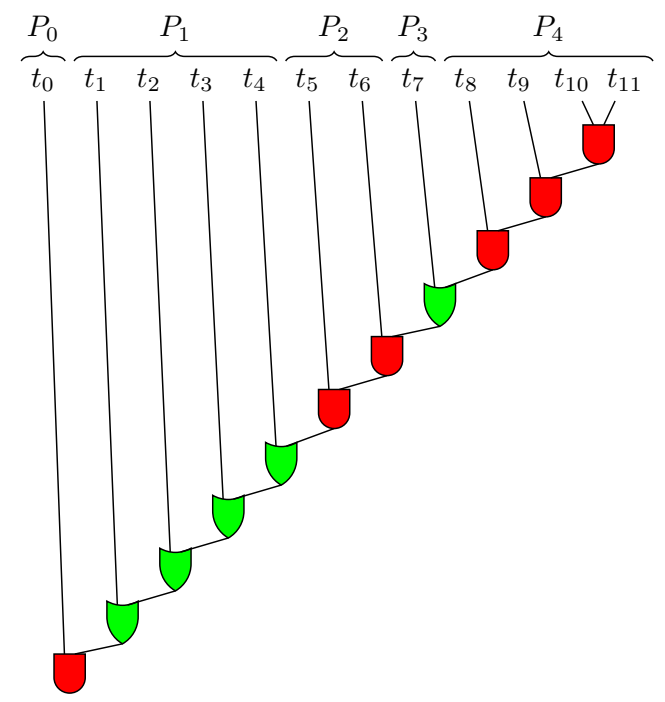

(a) A generalized AND-OR path.

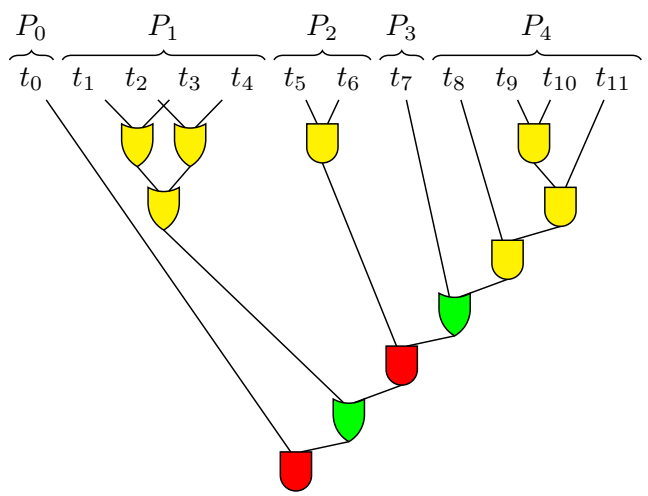

(b) Equivalent circuit after performing Huffman coding on the groups $P_{b}$.

Figure 4: Illustration of the proof of Theorem 30.

\section{More General Boolean Functions}

In this section, we extend Theorem 24 from AND-OR paths to similar functions that do not alternate between $\wedge$ and $\vee$ regularly, but arbitrarily.

Definition 29. We call a Boolean function of the form

$$
h\left(t, \circ_{1}, \ldots, \circ_{m-1}\right):=t_{0} \circ_{1}\left(t_{1} \circ_{2}\left(t_{2} \circ_{3}\left(\ldots \circ_{m-2}\left(t_{m-2} \circ_{m-1} t_{m-1}\right) \ldots\right)\right)\right)
$$

where $t=\left(t_{0}, \ldots, t_{m-1}\right)$ are Boolean input variables and for each $i \in\{1, \ldots, m-1\}$, the symbol $o_{i}$ denotes a two-input gate over the basis $\{\wedge, \vee\}$, a generalized And-Or path.

Theorem 30. Given Boolean input variables $t=\left(t_{0}, \ldots, t_{m-1}\right)$ and gates $\circ_{1}, \ldots, \circ_{m-1}$, there is a circuit realizing the generalized AND-OR path $h\left(t, \circ_{1}, \ldots, \circ_{m-1}\right)$ with delay at most

$$
\log _{2}(W)+\log _{2} \log _{2}(c+1)+\log _{2} \log _{2} \log _{2}(c+1)+5.3
$$

size at most $10(c+1) \log _{2}(c+1) \log _{2} \log _{2}(c+1)+m-c-1$ and maximum fanout at most $\log _{2}(c+1)+\log _{2} \log _{2}(c+1)+\log _{2} \log _{2} \log _{2}(c+1)+3.3$, where $c$ denotes the number of changes between $\wedge$ and $\vee$ or vice versa.

Proof. We first prove the delay bound. We partition the inputs $t_{0}, \ldots, t_{m-1}$ of $h$ into $c+1$ maximal groups $P_{0}, \ldots, P_{c}$ of consecutive inputs that feed the same kind of gate, see Figure 4 (a). We denote the common gate type of the gates fed by the inputs $P_{b}$ by $G_{b}$. Note that for each $b \in\{0, \ldots, c-1\}$, the group $P_{b}$ contains at least 1 input, while $P_{c}$ contains at least 2 inputs. 
For each $b \in\{0, \ldots, c\}$, we can build a symmetric binary $G_{b}$-tree on the inputs $P_{b}$ using Huffman coding. By Remark 8 , this yields a Boolean circuit $C_{b}$ with output $t_{b}^{\prime}$ with delay

$$
a\left(t_{b}^{\prime}\right)=\left\lceil\log _{2}\left(\sum_{t_{i} \in P_{b}} W\left(t_{i}\right)\right)\right]
$$

Denote the outputs of the circuits $C_{0}, \ldots, C_{c}$ by $t_{0}^{\prime}, \ldots, t_{c}^{\prime}$, respectively. Without loss of generality, we may assume that $\circ_{1}=\wedge$. Then, we can express $h\left(t, \circ_{1}, \ldots, \circ_{m-1}\right)$ as an AND-OR path as follows:

$$
h\left(t, \circ_{1}, \ldots, \circ_{m-1}\right)=g\left(t_{0}^{\prime}, \ldots, t_{c}^{\prime}\right)
$$

In Figure 4 (b), you can see the circuit arising from the generalized AND-OR Path in Figure 4 (a) in this way: The yellow gates are used for the circuits $C_{b}$ on the input groups $P_{b}$; and their outputs feed an AND-OR path drawn with red AND and green OR gates.

Write $W^{\prime}:=\sum_{b=0}^{c} W\left(t_{i}^{\prime}\right)$. Theorem 24 yields a circuit $C$ for $g\left(\left(t_{0}^{\prime}, \ldots, t_{c}^{\prime}\right)\right)$ and thus also for $h\left(t, \circ_{1}, \ldots, \circ_{m-1}\right)$ with delay at most

$$
\log _{2}\left(W^{\prime}\right)+\log _{2} \log _{2}(c+1)+\log _{2} \log _{2} \log _{2}(c+1)+4.3 .
$$

Note that the weight $W^{\prime}$ can be bounded by

$$
W^{\prime}=\sum_{b=0}^{c} 2^{a\left(t_{b}^{\prime}\right)} \stackrel{(29)}{\leq} \sum_{b=0}^{c} 2^{\log _{2}\left(\sum_{t_{i} \in P_{b}} W\left(t_{i}\right)\right)+1}=2 \sum_{b=0}^{c} \sum_{t_{i} \in P_{b}} W\left(t_{i}\right)=2 W(t) .
$$

Hence, the delay of $C$ stated in (31) can be bounded by

$$
\begin{array}{r}
\log _{2}\left(W^{\prime}\right)+\log _{2} \log _{2}(c+1)+\log _{2} \log _{2} \log _{2}(c+1)+4.3 \\
\leq \quad \log _{2}(W)+\log _{2} \log _{2}(c+1)+\log _{2} \log _{2} \log _{2}(c+1)+5.3,
\end{array}
$$

which finishes the proof of the delay bound.

For bounding the size of the arising circuit, note that for each $b \in\{0, \ldots, c\}$, the circuit $C_{b}$ has $\left|P_{b}\right|-1$ gates. Together with the size bound for the circuit realizing the AND-Or path (30) on $c+1$ inputs shown in Theorem 27, we obtain a total size of at most

$$
\begin{aligned}
& \sum_{b=0}^{c}\left(\left|P_{b}\right|-1\right)+10(c+1) \log _{2}(c+1) \log _{2} \log _{2}(c+1) \\
= & m-c-1+10(c+1) \log _{2}(c+1) \log _{2} \log _{2}(c+1) .
\end{aligned}
$$

Since in the circuits $P_{b}, b=0, \ldots, c+1$, every node has exactly one predecessor and each input of the AND-OR path (30) occurs only once, the maximum fanout occurs in the circuit for the And-Or path (30). Due to Theorem 27, this fanout is thus at most

$$
\log _{2}(c+1)+\log _{2} \log _{2}(c+1)+\log _{2} \log _{2} \log _{2}(c+1)+3.3 .
$$




\section{References}

[1] Richard Brent. On the addition of binary numbers. IEEE Transactions on Computers, 19(8):758-759, 1970.

[2] Richard P. Brent and H.-T. Kung. A regular layout for parallel adders. IEEE Transactions on Computers, 31(3):260-264, 1982.

[3] Beate Commentz-Walter. Size-depth tradeoff in monotone Boolean formulae. Acta Informatica, 12(3):227-243, 1979.

[4] Beate Commentz-Walter and Juergen Sattler. Size-depth tradeoff in non-monotone Boolean formulae. Acta Informatica, 14(3):257-269, 1980.

[5] Martin C. Golumbic. Combinatorial merging. IEEE Transactions on Computers, 25(11):1164-1167, 1976.

[6] Mikhail I. Grinchuk. Sharpening an upper bound on the adder and comparator depths. Journal of Applied and Industrial Mathematics, 3(1):61-67, 2009.

[7] Stephan Held and Sophie Spirkl. Fast prefix adders for non-uniform input arrival times. Algorithmica, 77(1):287-308, 2017.

[8] David A. Huffman. A method for the construction of minimum-redundancy codes. Proceedings of the Institute of Radio Engineers, 40(9):1098-1101, 1952.

[9] Valerii M. Khrapchenko. Asymptotic estimation of addition time of parallel adder. Syst. Theory Res., 19:105-122, 1970.

[10] Valerii M. Khrapchenko. On possibility of refining bounds for the delay of a parallel adder. Journal of Applied and Industrial Mathematics, 2(2):211-214, 2008.

[11] Peter M. Kogge and Harold S. Stone. A parallel algorithm for the efficient solution of a general class of recurrence equations. IEEE Transactions on computers, 100(8):786-793, 1973.

[12] Leon Gordon Kraft. A device for quantizing, grouping, and coding amplitude-modulated pulses. PhD thesis, Massachusetts Institute of Technology, 1949.

[13] Richard E. Ladner and Michael J. Fischer. Parallel prefix computation. Journal of the ACM (JACM), 27(4):831-838, 1980.

[14] Dieter Rautenbach, Christian Szegedy, and Jürgen Werber. Asymptotically optimal Boolean circuits for functions of the form $g_{n-1}\left(g_{n-2}\left(\ldots g_{3}\left(g_{2}\left(g_{1}\left(x_{1}, x_{2}\right), x_{3}\right), x_{4}\right) \ldots, x_{n-1}\right), x_{n}\right)$ given input arrival times. Research Institute for Discrete Mathematics, University of Bonn, 2003.

[15] Dieter Rautenbach, Christian Szegedy, and Jürgen Werber. Delay optimization of linear depth Boolean circuits with prescribed input arrival times. Journal of Discrete Algorithms, $4(4): 526-537,2006$.

[16] John E. Savage. Models of Computation, volume 136. Addison-Wesley Reading, MA, 1998.

[17] Jack Sklansky. Conditional-sum addition logic. IRE Transactions on Electronic computers, (2):226-231, 1960 .

[18] Sophie Spirkl. Boolean circuit optimization. Master's thesis, University of Bonn, 2014. 
[19] Jürgen Werber. Logic Restructuring for Timing Optimization in VLSI Design. PhD thesis, University of Bonn, 2007.

[20] Jürgen Werber, Dieter Rautenbach, and Christian Szegedy. Timing optimization by restructuring long combinatorial paths. In Proceedings of the 2007 IEEE/ACM International Conference on Computer-Aided Design, pages 536-543. IEEE Press, 2007.

[21] Reto Zimmermann. Binary Adder Architectures for Cell-Based VLSI and Their Synthesis. PhD thesis, Swiss Federal Institute of Technology, Zurich (ETH), 1998. 